\title{
Stability analysis of non-autonomous reaction-diffusion systems: the effects of growing domains
}

\author{
Anotida Madzvamuse • Eamonn A. Gaffney • \\ Philip K. Maini
}

Received: 18 February 2009 / Revised: 31 July 2009 / Published online: 29 August 2009 (C) Springer-Verlag 2009

\begin{abstract}
By using asymptotic theory, we generalise the Turing diffusively-driven instability conditions for reaction-diffusion systems with slow, isotropic domain growth. There are two fundamental biological differences between the Turing conditions on fixed and growing domains, namely: (i) we need not enforce cross nor pure kinetic conditions and (ii) the restriction to activator-inhibitor kinetics to induce pattern formation on a growing biological system is no longer a requirement. Our theoretical findings are confirmed and reinforced by numerical simulations for the special cases of isotropic linear, exponential and logistic growth profiles. In particular we illustrate an example of a reaction-diffusion system which cannot exhibit a diffusively-driven instability on a fixed domain but is unstable in the presence of slow growth.
\end{abstract}

Keywords Convection-reaction-diffusion systems - Turing diffusively-driven instability · Pattern formation - Growing domains asymptotic theory ·

Domain-induced diffusively-driven instability

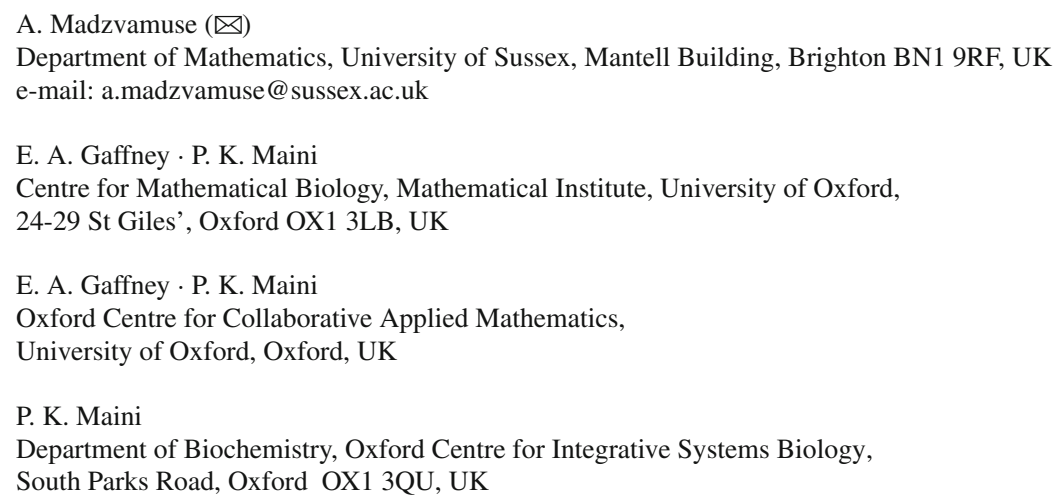




\section{Introduction}

Reaction-diffusion equations (RDEs) have been widely proposed as plausible models of pattern generation processes Murray (2002). On fixed domains, Turing (1952) derived the conditions under which a linearised reaction-system admits a linearly stable spatially homogeneous steady state in the absence of diffusion and yet, becomes unstable under appropriate conditions in the presence of diffusion to yield a spatially varying inhomogeneous steady state. This process is now well-known as diffusively-driven instability and is of particular interest in developmental biological pattern formation as a means of initiating self organisation from a virtually homogeneous background. Turing patterns were first observed by Castets et al. (1990) in a chloride-ionic-malonic-acid (CIMA) reaction and Ouyang and Swinney (1991) were the first to observe a Turing instability from a spatially uniform state to a patterned state. Although controversial in a biological context for many years, recent experimental findings suggest this may be a mechanism for the formation of repeated structures in skin organ formation (Sick et al. 2006; Maini et al. 2006) and zebrafish mesoderm cell fates (Solnica-Krezel 2003).

On fixed domains, the properties of the autonomous Turing diffusively-driven instability conditions require that the reaction kinetics should be of activator-inhibitor form with the inhibitor diffusing faster, typically much faster, than the activator. This gives rise to the standard paradigm of pattern formation via short-range activation and long-range inhibition. Most applications of Turing's theory have assumed fixed domains; in the context of developmental biology, this requires the tacit assumption that pattern forming processes occur on a different timescale to that of domain growth. However, while growth is usually anticipated to occur at a slower rate than other biological processes, it nonetheless has an important dynamical role. For example, slow domain growth will typically dictate the nature of the pattern that evolves as the domain grows leading to a much greater robustness of pattern compared to the array of patterning that can take place on a fixed domain. This is illustrated by Kondo and Asai (1995) who predicted mode doubling in pigmentation patterns of the angelfish Pomacanthus as it grows. The juvenile Pomacanthus has three vertical stripes; once the fish grows to twice its original size, new stripes emerge between the original stripes so that the original wavelength is maintained. In contrast to the case of a fixed domain, numerous different stripe and spot patterns occur depending on perturbations of the initial conditions for the model. Further examples of studies of RDEs illustrating the role of domain growth can be found in papers by Varea et al. (1999), Chaplain et al. (2001), Liaw et al. (2001), Painter et al. (1999), Crampin et al. (2002, 1999), Oster and Bressloff (2006), Madzvamuse et al. (2003, 2005), Madzvamuse (2005) and for a review see Plaza et al. (2004).

In particular, the latter presented a framework to investigate the role of curvature and growth in pattern formation and selection via the Turing instability. The corresponding Turing analysis on growing domains was not attempted. Instead, they analysed 
equations that allow the separation of the geometrical spatial effects from those due to domain growth with the assumption of isotropic linear growth. In all their simulations they observed that the selection of the final pattern was dictated by the interplay of the curvature and domain growth given fixed model parameter values. Transient patterns were shown to be robustly selected due to the effects of either curvature and/or domain growth, in complete agreement with previous results obtained in computational studies by Crampin et al. (1999, 2002) and Madzvamuse et al. (2003), Madzvamuse (2005).

As the first step in considering a Turing instability analysis, it can be shown that RDEs on a growing domain can be transformed into a RDEs on a fixed domain, but with time-dependence in the diffusion and dilution terms Crampin et al. (2002). These non-autonomous terms however typically invalidate standard linear stability analysis via plane wave decompositions, even with the common simplification that the domain growth is assumed to be isotropic, whereby the domain expands at the same rate in all directions at all times. From a mathematical point of view, stability conditions on fixed domains are typically derived from the calculation of the eigenvalues of a time-independent matrix governing the dynamics of perturbations in the linear regime. Slow growth induces initially small but time-dependent and cumulative changes in such matrices. However, eigenvalues can, in general, be very sensitive to matrix perturbations (Golub and Van Loan 1996), and therefore it is unclear a priori whether the standard conditions for a diffusively-driven instability will transfer even in the presence of small growth.

Despite the above-mentioned studies and the simplifying assumption of isotropy, there is only limited analytical work investigating the impact domain growth has on pattern formation (see, for example Plaza et al. (2004)). Hence, this paper aims to derive and present the Turing diffusively-driven instability conditions for RDEs on continuously deforming growing domains restricted to the case of slow growth only. In most biological systems that involve domain growth, such growth occurs slowly and therefore our assumption is biologically relevant as detailed below. Linearising the model equations around a time-dependent solution $\boldsymbol{u}_{S}(t)$ we show that $\boldsymbol{u}_{S}(t)$ is a solution of a non-autonomous system of ordinary differential equations. Using asymptotic theory, we further derive and state the conditions that generalise the classic Turing parameter space inequalities for the case of slow growing domains and we show that these conditions are a function of the model parameters in the reaction terms and the growth dynamics. Two fundamental biological differences between the Turing conditions on fixed and growing domains are:

(i) One need not enforce cross nor pure kinetic conditions.

(ii) The restriction to activator-inhibitor kinetics to induce pattern formation on a growing biological system is no longer a requirement.

In particular we observe below that an activator-activator mechanism may give rise to what we will term domain-growth-induced Turing instability. Similarly, in the presence of growth, short-range inhibition, long-range activation can also generate a diffusively-driven instability. Such mechanisms for pattern formation are impossible on fixed domains because of the stability constraints imposed. Finally, to illustrate our theoretical findings, we compute numerical results for the special cases of isotropic linear, exponential and logistic growth profiles in one spatial dimension. 
Hence, our paper is organised as follows: in Sect. 2 we present the model equations derived from first principles on a continuously deforming domain. The resultant model equations are then transformed, using a Lagrangian coordinate system, into a nonconservative formulation. The derivation of stability conditions for diffusively-driven instabilities using asymptotic theory for the case of slow growth is presented in Sect. 3. To illustrate our theoretical findings, two examples are presented as shown in Sect. 4. The first example is an activator-depleted model known to satisfy the cross/pure kinetics conditions (Gierer and Meinhardt 1972; Prigogine and Lefever 1968; Schnakenberg 1979). The second example consists of reaction kinetics which do not satisfy any of the cross/pure kinetics conditions. Our aim is to show that the effects of incorporating domain growth does not necessarily restrict one to consider only a short range activator-long range inhibition mechanism, in distinct contrast to pattern formation on fixed domains. Finally, in Sect. 5 we summarise, discuss and interpret the results of our research and conclude.

\section{Reaction-diffusion systems on continuously growing domains}

Let $\Omega_{t} \subset \mathbb{R}^{m}$ ( $\left.m=1,2,3\right)$ be a simply connected bounded continuously deforming volume at time $t \in I=\left[0, t_{F}\right], t_{F}>0$ and $\partial \Omega_{t}$ be the surface boundary of the continuously changing volume. Also let $\boldsymbol{u}=(u(\boldsymbol{x}(t), t), v(\boldsymbol{x}(t), t))^{T}$ be a vector of two chemical concentrations at position $\boldsymbol{x}=(x(t), y(t), z(t)) \in \Omega_{t} \subset \mathbb{R}^{m}$. The evolution equations for reaction-diffusion on a growing domain are readily obtained from conservation of mass in an elemental volume using Reynolds transport theorem. RDEs of the type studied for pattern formation generally exclude cross-diffusion and are only coupled through the reaction terms. The growth of the domain $x \in \Omega_{t}$ with boundary $\partial \Omega_{t}$ generates a flow velocity $\boldsymbol{a}(\boldsymbol{x}, t)$. The non-dimensionalised governing equations take the form (Crampin et al. 2002; Madzvamuse et al. 2003; Madzvamuse 2005)

$$
\left\{\begin{array}{c}
\underbrace{u_{t}}_{\text {Rate of change of } u}+\underbrace{\nabla \cdot(\boldsymbol{a} u)}_{\text {growth terms }}=\underbrace{\nabla^{2} u}_{\text {diffusion }}+\underbrace{\gamma f(u, v)}_{\text {nonlinear reaction terms }} \\
\underbrace{v_{t}}_{\text {Rate of change of } v}+\underbrace{\nabla \cdot(\boldsymbol{a} v)}_{\text {growth terms }}=\underbrace{d \nabla^{2} v}_{\text {diffusion }}+\underbrace{\gamma g(u, v)}_{\text {nonlinear reaction terms }}
\end{array} .\right.
$$

Here, $f(u, v)$ and $g(u, v)$ represent nonlinear reaction kinetics, $d$ is a constant ratio of the diffusion coefficients and $\gamma$ is a scaling parameter. These equations are supplemented with initial conditions

$$
\boldsymbol{u}=\boldsymbol{u}_{0}(\boldsymbol{x}), \quad \text { for } \boldsymbol{x} \in \Omega_{0} \text { at } t=0,
$$

where $\boldsymbol{u}_{0}(\boldsymbol{x})$ is a positive bounded vector function. We typically consider either zeroflux boundary conditions,

$$
(\boldsymbol{n} \cdot \nabla) \boldsymbol{u}=\mathbf{0}, \quad \boldsymbol{x} \in \partial \Omega_{t} \text { and } t \geq 0
$$


where $\boldsymbol{n}$ is the unit normal vector to the surface $\partial \Omega_{t}$ or homogeneous Dirichelet conditions

$$
\boldsymbol{u}=\mathbf{0} \quad \boldsymbol{x} \in \partial \Omega_{t} \text { and } t \geq 0
$$

which correspond to sinks at the domain boundaries.

In compact-vector form we can write (1) as:

$$
\boldsymbol{u}_{t}+\nabla \cdot(\boldsymbol{a}: \boldsymbol{u}-\boldsymbol{D} \nabla \boldsymbol{u})=\gamma \boldsymbol{F}(\boldsymbol{u})
$$

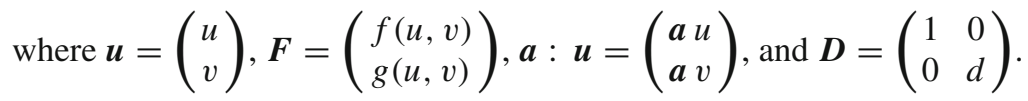

\subsection{A Lagrangian coordinate system}

We consider a new spatial coordinate system $\xi$ via the bijective mapping

$$
\xi_{i}=\xi_{i}(\boldsymbol{x}, t), \quad x_{q}=x_{q}(\xi, t)
$$

where $\boldsymbol{\xi} \in \Omega_{0}$ and $\boldsymbol{x} \in \Omega_{t}$. From now onwards, all lowercase Latin subscripts (excluding $u, v, h_{*}$ ) index spatial coordinates. We adopt the usual summation convention for such indices whereby repetition denotes an implicit summation over the number of spatial dimensions. From the chain rule we immediately have the identities

$$
\frac{\partial \xi_{i}}{\partial t}+\frac{\partial \xi_{i}}{\partial x_{q}} \frac{\partial x_{q}}{\partial t}=0 \text { and } \frac{\partial \xi_{i}}{\partial x_{q}} \frac{\partial x_{q}}{\partial \xi_{s}}=\delta_{i s}
$$

We also have that Eq. (2) transforms to

$$
\left.\frac{\partial \boldsymbol{u}}{\partial t}\right|_{\boldsymbol{\xi}}+\frac{\partial \xi_{i}}{\partial t} \frac{\partial \boldsymbol{u}}{\partial \xi_{i}}+\frac{\partial \xi_{r}}{\partial x_{q}} \frac{\partial}{\partial \xi_{r}}\left(a_{q} \boldsymbol{u}\right)=\boldsymbol{D} \frac{\partial \xi_{i}}{\partial x_{j}} \frac{\partial}{\partial \xi_{i}}\left(\frac{\partial \xi_{p}}{\partial x_{j}} \frac{\partial \boldsymbol{u}}{\partial \xi_{p}}\right)+\gamma \boldsymbol{F}(\boldsymbol{u}),
$$

where $\boldsymbol{D}=\operatorname{diag}(1, d)$. This simplifies to

$$
\begin{aligned}
& \left.\frac{\partial \boldsymbol{u}}{\partial t}\right|_{\boldsymbol{\xi}}+\left[\left[\frac{\partial \xi_{i}}{\partial t}+\frac{\partial \xi_{i}}{\partial x_{q}} a_{q}\right] \boldsymbol{I}-\frac{\partial^{2} \xi_{i}}{\partial x_{q} \partial x_{q}} \boldsymbol{D}\right] \frac{\partial \boldsymbol{u}}{\partial \xi_{i}}+\frac{\partial \xi_{r}}{\partial x_{q}} \frac{\partial a_{q}}{\partial \xi_{r}} \boldsymbol{u} \\
& =\boldsymbol{D} \frac{\partial \xi_{i}}{\partial x_{j}} \frac{\partial \xi_{p}}{\partial x_{j}} \frac{\partial^{2} \boldsymbol{u}}{\partial \xi_{i} \partial \xi_{p}}+\gamma \boldsymbol{F}(s \boldsymbol{u}) .
\end{aligned}
$$

The bijectivity of the mapping ensures that initial conditions can be readily written with respect to $\xi$ and that homogeneous boundary conditions, whether Neumann or Dirichlet, are also inherited unchanged. On choosing a mapping which obeys

$$
\left.\frac{\partial x_{q}}{\partial t}\right|_{\xi}=a_{q}
$$


we have, via Eq. (4), that

$$
\left.\frac{\partial \boldsymbol{u}}{\partial t}\right|_{\boldsymbol{\xi}}-\left[\frac{\partial^{2} \xi_{i}}{\partial x_{q} \partial x_{q}}\right] \boldsymbol{D} \frac{\partial \boldsymbol{u}}{\partial \xi_{i}}+\frac{\partial \xi_{r}}{\partial x_{q}} \frac{\partial a_{q}}{\partial \xi_{r}} \boldsymbol{u}=\boldsymbol{D} \frac{\partial \xi_{i}}{\partial x_{j}} \frac{\partial \xi_{p}}{\partial x_{j}} \frac{\partial^{2} \boldsymbol{u}}{\partial \xi_{i} \partial \xi_{p}}+\gamma \boldsymbol{F}(\boldsymbol{u}) .
$$

A standard choice is

$$
\left.\frac{\partial}{\partial t}[\boldsymbol{x}(\xi, t)]\right|_{\xi}=a(x, t), \quad x(\xi, 0)=\xi
$$

which is consistent with Eq. (5) and ensures that $\xi$ corresponds to a Lagrangian coordinate system.

\subsubsection{Spatially linear, isotropic growth}

Hereafter, we assume without loss of generality that $\boldsymbol{\xi}$ is a Lagrangian coordinate system and thus that Eq. (7) is satisfied. We wish to consider the simplest, non-trivial, form of growth and thus consider the expansion

$$
a_{k}(\boldsymbol{x}, t)=v_{k}(t)+A_{k p}(t) x_{p}+S_{k p}(t) x_{p}+B_{k p q}(t) x_{p} x_{q}+\cdots
$$

where $A_{k p}(t)$ is antisymmetric and $S_{k p}(t)$ is symmetric. Clearly, $v_{k}(t)$ corresponds to a rigid body translation and $A_{k p}(t)$ to a rigid body rotation. Because we wish to consider growth rather than rigid body movements we set $v_{k}(t)$ and $A_{k p}(t)$ to zero. To consider the simplest form of growth, we set higher order terms such as $B_{k p q}(t)$ to zero and thus consider

$$
a_{k}(\boldsymbol{x}, t)=S_{k p}(t) x_{p}
$$

with $S_{k p}(t)$ symmetric, which we define to be spatially linear growth. Rather than deal with this general case we consider the simpler case of isotropic growth, where $S_{k p}(t)$ is proportional to the identity matrix. Thus

$$
S_{k p}(t)=S(t) \delta_{k p}
$$

where $S(t)$ represents the expansion (or contraction) rate of the domain and $\delta_{k p}$ is the Kronecker delta. Hence Eq. (6) simplifies to

$$
\frac{\partial \boldsymbol{u}}{\partial t}+h(t) \boldsymbol{u}=\frac{1}{\varphi^{2}(t)} \boldsymbol{D} \frac{\partial^{2} \boldsymbol{u}}{\partial \xi_{i} \partial \xi_{i}}+\gamma \boldsymbol{F}(\boldsymbol{u})
$$

where $\frac{\partial^{2} \boldsymbol{u}}{\partial \xi_{i} \partial \xi_{i}}=\nabla^{2} \boldsymbol{u}$ in multi-dimensions and

$$
h(t)=m S(t), \quad \varphi(t)=\exp \left[\int_{0}^{t} d q S(q)\right] \geq 0,
$$


with $m$ denoting the number of spatial dimensions. It should be noted that:

1. In one dimension, spatially linear growth (with no further assumptions) is of this form; the assumption of isotropy required in higher dimensions is redundant. Our analysis for the rest of the paper focuses on the model equations (8) and (9).

2. The model equation (8) is in non-conservation form. The conservative formulation required for the numerical simulations below can be easily deduced by using an arbitrary Lagrangian-Eulerian reference frame (Madzvamuse 2008).

It is worth pointing out that numerical solutions corresponding to Eq. (8) have been extensively studied in papers by Crampin et al. (2002, 1999), Plaza et al. (2004), Madzvamuse (2005), and Madzvamuse and Maini (2007).

\subsubsection{Timescales}

For a biological system, growth is typically driven by cell division, and thus occurs on the timescale of the cell cycle duration, which is typically $24 \mathrm{~h}$ or greater. In contrast, the biochemical reaction kinetics are typically considered to occur on a much faster time scale of seconds to minutes, and are typically slave to the diffusive dynamics. The length scale associated with a volume of $10^{4}$ to $10^{6}$ cells is about $2 \times 10^{-4} \mathrm{~m}$ to $10^{-3} \mathrm{~m}$. Given a diffusion coefficient of between $10^{-10} \mathrm{~m}^{2} \mathrm{~s}^{-1}$ to $10^{-9} \mathrm{~m}^{2} \mathrm{~s}-1$, the diffusive timescale is about $40 \mathrm{~s}$ to $170 \mathrm{~min}$. We will take advantage of this difference in timescales in our analysis. With $T_{g}$ denoting the growth timescale, and $T_{d y n}$ denoting the maximum of the diffusive timescale and the biochemical kinetics timescale, we will have occasion to utilise the small parameter

$$
\epsilon \stackrel{\text { def }}{=} \frac{T_{d y n}}{T_{g}} \ll 1 ;
$$

and therefore we will generally neglect $O\left(\epsilon^{2}\right)$ corrections. For the above range of estimates, we have $\epsilon \in\left[5 \times 10^{-4}, 0.12\right]$.

\section{Domain-growth-induced diffusively-driven instability analysis}

\subsection{Definitions}

In the following, we consider the diffusively-driven instability analysis of spatially linear, isotropic growth. Given Eqs. (8) and (9) do not always admit a uniform steady state, we first of all consider the spatially independent solution defined as follows:

Definition 1 Let $\boldsymbol{u}_{S}(t)$ be a spatially independent solution of (8). Clearly $\boldsymbol{u}_{S}(t)$ is a solution of the non-autonomous ordinary differential vector equation

$$
h(t) \boldsymbol{u}_{S}+\frac{\partial \boldsymbol{u}_{S}}{\partial t}=\gamma \boldsymbol{F}\left(\boldsymbol{u}_{S}\right), \quad \text { given } \boldsymbol{u}_{S}(0)=\boldsymbol{u}_{S}^{0} \in \mathbb{R}
$$


Definition 2 (Diffusively-Driven Instability on Growing Domains) Diffusivelydriven instability (or Turing instability) on growing domains occurs when a spatially independent vector function $\boldsymbol{u}_{S}(t)$, linearly stable in the absence of diffusion, goes unstable when diffusion is present.

\subsection{Non-autonomous behaviour of the linearised system}

To proceed in investigating the possibility of a diffusively-driven instability in the presence of growth, we work in the Lagrangian coordinate system and expand $\boldsymbol{u}(\boldsymbol{\xi}, t)$ about the spatially independent solution $\boldsymbol{u}_{S}(t)$. Thus we substitute

$$
\boldsymbol{u}(\boldsymbol{\xi}, t)=\boldsymbol{u}_{S}(t)+\eta \boldsymbol{w}(\boldsymbol{\xi}, t) \quad \text { with } \eta \ll 1,
$$

into Eqs. (8) and (9). On neglect of $O\left(\eta^{2}\right)$ and higher order terms, we obtain

$$
\frac{\partial \boldsymbol{w}}{\partial t}+h(t) \boldsymbol{w}=\frac{1}{\varphi^{2}(t)} \boldsymbol{D} \frac{\partial^{2} \boldsymbol{w}}{\partial \xi_{i} \partial \xi_{i}}+\left.\gamma \boldsymbol{J}_{\boldsymbol{F}}(t)\right|_{\boldsymbol{u}_{S}} \boldsymbol{w}
$$

where $\boldsymbol{J}_{\boldsymbol{F}}(t)$ is the Jacobian matrix corresponding to the non-linear reaction kinetics which is evaluated at $\boldsymbol{u}_{S}(t)$. We define $\psi_{K}(\boldsymbol{\xi})$ to be the time independent eigenmode of the transport operator satisfying

$$
\left\{\begin{array}{l}
\frac{\partial^{2} \psi_{K}}{\partial \xi_{i} \partial \xi_{i}}=-K^{2} \psi_{K}, \quad \xi \in \Omega_{0}, \\
n_{j} \frac{\partial \psi_{K}}{\partial \xi_{j}}=0, \quad \xi \in \partial \Omega_{0},
\end{array}\right.
$$

where $n_{j}$ denotes components of the outward pointing surface normal of $\partial \Omega_{0}$. A standard analysis shows that the eigenvalue must be real and negative, and thus without loss of generality, we write it as $-K^{2}$ in the above; we similarly have that eigenmodes of distinct eigenvalues are orthogonal. Furthermore, note that the domain $\Omega_{0}$ is time-independent because $\boldsymbol{\xi}$ is a Lagrangian coordinate system.

Observe that in the one-dimensional case with homogeneous Neumann Boundary conditions and an initial domain length $L$, the modes are given by

$$
\psi_{K}(\xi)=A_{K} \cos (K \xi)
$$

where $K=\frac{n \pi}{L}, n= \pm 1, \pm 2 \ldots$ are discrete wavenumbers and $A_{K}$ is constant. Analogous definitions apply for homogeneous Dirichlet boundary conditions. Without loss of generality, the constant $A_{K}$ is typically determined by demanding othonormality in addition to orthogonality of the eigenmodes. Below, there is an implicit assumption that the eigenmodes are complete though we note that with simple geometries, completeness with respect to the $L_{2}$-norm is inherited from the properties of Fourier series. 
We proceed by expanding $\boldsymbol{w}$ in terms of the eigenmodes

$$
\boldsymbol{w}(\boldsymbol{\xi}, t)=\sum_{K} \boldsymbol{w}_{K}(\boldsymbol{\xi}, t)=\sum_{K} \boldsymbol{a}_{K}(t) \psi_{K}(\boldsymbol{\xi})
$$

Linearity and the orthogonality of the eigenmodes allow each mode to be considered separately, which is a substantial simplification. In particular, growth of any one of the $\boldsymbol{a}_{K}(t)$ with time is sufficient to drive the full solution away from the time-dependent solution $\boldsymbol{u}_{S}(t)$. Similarly, if all the $\boldsymbol{a}_{K}(t)$ decay with time then a sufficiently small perturbation from the time-dependent solution $\boldsymbol{u}_{S}(t)$ will decay, at least within the resolution of linear theory predictions.

To proceed, we substitute, for each $K$, a mode $\boldsymbol{w}_{K}=\boldsymbol{a}_{K}(t) \psi_{K}(\boldsymbol{\xi})$ from expansion (14) into the linearised equations (12) to find

$$
\left[\left(\dot{\boldsymbol{a}}_{K}+h(t) \boldsymbol{a}_{K}\right)+\frac{K^{2}}{\varphi^{2}(t)} \boldsymbol{D} \boldsymbol{a}_{K}-\left.\gamma \boldsymbol{J}_{\boldsymbol{F}}(t)\right|_{\boldsymbol{u}_{S}} \boldsymbol{a}_{K}\right] \psi_{K}=\mathbf{0} .
$$

Writing

$$
\boldsymbol{a}_{K}(t)=\boldsymbol{b}_{K}(t) q(t),
$$

where $q(t)=\exp \left[-\int_{t_{0}}^{t} h(\tau) d \tau\right]$ and $t_{0}$ is the time the perturbation is applied, we have

$$
\left[\dot{\boldsymbol{b}}_{K}+\frac{K^{2}}{\varphi^{2}(t)} \boldsymbol{D} \boldsymbol{b}_{K}-\left.\gamma \boldsymbol{J}_{\boldsymbol{F}}(t)\right|_{\boldsymbol{u}_{S}} \boldsymbol{b}_{K}\right] \psi_{K} q(t)=\mathbf{0} .
$$

For the autonomous case, it would be standard at this point to substitute $\boldsymbol{b}_{k}(t)=e^{\alpha t} \boldsymbol{b}_{k}^{0}$ in the above to determine an eigenvalue equation for $\alpha$. This is no longer appropriate with non-autonomy, despite its previous use in the literature (Gjorgjieva and Jacobsen 2007 ), since this approach implicity requires the contradiction that $\boldsymbol{b}_{k}^{0}$, which is time independent, is an eigenvector of a time-dependent matrix. Instead we proceed as follows. One immediately has from Eq. (16) that

$$
\frac{d \boldsymbol{b}_{K}}{d t}=\boldsymbol{M}_{K}(t) \boldsymbol{b}_{K}, \quad \text { where } \boldsymbol{M}_{K}(t) \stackrel{\text { def }}{=}\left[-\frac{K^{2}}{\varphi^{2}(t)} \boldsymbol{D}+\left.\gamma \quad \boldsymbol{J}_{\boldsymbol{F}}(t)\right|_{\boldsymbol{u}_{S}}\right]
$$

Given initial conditions $\boldsymbol{b}_{K}\left(t_{0}\right)$ at time $t_{0}$ we seek a solution at time $t>t_{0}$ of the form

$$
\boldsymbol{b}_{K}(t)=\exp \left[\int_{t_{0}}^{t} d s \lambda_{K}^{*}(s)\right] \boldsymbol{c}_{K}(t)
$$


where $\lambda_{K}^{*}(t)$ is either the largest time-dependent real eigenvalue of $\boldsymbol{M}_{K}(t)$ or the real part of one of the time-dependent complex conjugate eigenvalues. ${ }^{1}$ From Eqs. (17) and (18) we have

$\frac{d \boldsymbol{c}_{K}}{d t}=\boldsymbol{Q}_{K}(t) \boldsymbol{c}_{K}$, where $\boldsymbol{Q}_{K}(t) \stackrel{\text { def }}{=}\left[\boldsymbol{M}_{K}(t)-\lambda_{K}^{*}(t) \boldsymbol{I}\right]$, with $\boldsymbol{c}_{K}\left(t_{0}\right)=\boldsymbol{b}_{K}\left(t_{0}\right)$.

3.3 Analysis of solutions of non-autonomous systems of ordinary differential equations

\subsection{1 $\mathrm{M}_{K}(t)$ has real, distinct eigenvalues}

One eigenvalue of $\boldsymbol{Q}_{K}(t)$ is zero and the other is negative. Let $\boldsymbol{e}_{K}^{0}(t)$ be the unit eigenvector associated with the zero eigenvalue and let $\boldsymbol{e}_{K}^{1}(t)$ be the unit eigenvector associated with the negative eigenvalue, such that the angle between $\boldsymbol{e}_{K}^{0}(t)$ and $\boldsymbol{e}_{K}^{1}(t)$ is acute. These eigenvectors are distinct and thus a spanning set; hence one can write

$$
\boldsymbol{c}_{K}(t)=\alpha_{K}(t) \boldsymbol{e}_{K}^{0}(t)+\beta_{K}(t) \boldsymbol{e}_{K}^{1}(t), \quad \text { and } \quad\left\|\boldsymbol{c}_{K}(t)\right\|=\sqrt{\alpha_{K}^{2}(t)+\beta_{K}^{2}(t)} .
$$

The above norm is only the standard $L_{2}$-norm if $\boldsymbol{e}_{K}^{0}(t)$ and $\boldsymbol{e}_{K}^{1}(t)$ are perpendicular, which need not be true; nonetheless, $\|\cdot\|$ satisfies all the axioms required of a norm. For a matrix $Q$, the induced matrix norm is

$$
\|\boldsymbol{Q}\|=\sup _{\|\boldsymbol{x}\|=1}\|\boldsymbol{Q} \boldsymbol{x}\|
$$

and the Lozinskii measure is (Connell McCluskey 2005)

$$
\mu(\boldsymbol{Q})=\lim _{h \rightarrow 0} \frac{\|\boldsymbol{I}+h \boldsymbol{Q}\|-1}{h} .
$$

Noting $\|\boldsymbol{Q} \boldsymbol{x}\| \leq\|\boldsymbol{Q}\|\|\boldsymbol{x}\|$, for any matrix $\boldsymbol{Q}$, and vector $\boldsymbol{x}$, a solution of Eq. (19) satisfies

$$
\begin{aligned}
\frac{d}{d t}\left\|\boldsymbol{c}_{K}(t)\right\| & =\lim _{h \rightarrow 0} \frac{\left\|\boldsymbol{c}_{K}(t+h)\right\|-\left\|\boldsymbol{c}_{K}(t)\right\|}{h} \\
& =\lim _{h \rightarrow 0} \frac{\left\|\boldsymbol{c}_{K}(t)+h \boldsymbol{Q}_{K}(t) \boldsymbol{c}_{K}(t)\right\|-\left\|\boldsymbol{c}_{K}(t)\right\|}{h} \\
& \leq \lim _{h \rightarrow 0} \frac{\left\|\boldsymbol{I}+h \boldsymbol{Q}_{K}(t)\right\|\left\|\boldsymbol{c}_{K}(t)\right\|-\left\|\boldsymbol{c}_{K}(t)\right\|}{h}=\mu\left(\boldsymbol{Q}_{K}(t)\right)\left\|\boldsymbol{c}_{K}(t)\right\| .
\end{aligned}
$$

\footnotetext{
1 Should the eigenvalues of $\boldsymbol{M}_{K}(s)$ change from real to complex either side of time $s_{*} \in\left[t_{0}, t\right]$ we note that our analysis is not valid at $s_{*}$ just as the usual Turing analysis is not valid for eigenvalues with zero real part; one can consider times either side of $s_{*}$ with the analysis below.
} 
Hence

$$
\left\|\boldsymbol{c}_{K}(t)\right\| \leq\left\|\boldsymbol{c}_{K}(0)\right\| \exp \left[\int_{t_{0}}^{t} d s \mu\left(\boldsymbol{Q}_{K}(s)\right)\right]=\left\|\boldsymbol{c}_{K}(0)\right\|
$$

noting that a matrix with zero and negative eigenvalues has a zero Lozinskii measure. Thus a solution to Eq. (19) does not grow in time.

We now show that for $t$ fixed, with $\Delta t=t-t_{0} \sim T_{d y n}$, we can also find at least one solution of (19) which does not decay on neglect of $O\left(\epsilon^{2}\right)$ corrections; not surprisingly this corresponds to an initial condition along the direction of the zero eigenvector. From a Picard iteration we have

$$
\boldsymbol{c}_{K}(t)=\boldsymbol{G}_{K}\left(t, t_{0}\right) \boldsymbol{c}_{K}\left(t_{0}\right)
$$

where

$$
\boldsymbol{G}_{K}\left(t, t_{0}\right)=\boldsymbol{I}+\sum_{n=1}^{\infty} \int_{t_{0}}^{t} d t_{1} \int_{t_{0}}^{t_{1}} d t_{2} \ldots \int_{t_{0}}^{t_{n-1}} d t_{n} \boldsymbol{Q}_{K}\left(t_{1}\right) \boldsymbol{Q}_{K}\left(t_{2}\right) \ldots \boldsymbol{Q}_{K}\left(t_{n}\right)
$$

The existence of $\boldsymbol{G}_{K}\left(t, t_{0}\right)$ is guaranteed from Picard's theorem given the components of $\boldsymbol{Q}_{K}(t)$ are bounded to ensure that $\boldsymbol{Q}_{K}(t) \boldsymbol{c}_{K}(t)$ is Lipschitz. With "dot" denoting a time derivative, we have

$$
\dot{\boldsymbol{Q}}_{K} \sim O\left(\frac{1}{T_{\text {growth }}} \boldsymbol{Q}_{K}\right) \sim O\left(\frac{\epsilon}{T_{\text {dyn }}} \boldsymbol{Q}_{K}\right)
$$

since the change in the matrix $Q$ is driven by growth. We set the initial condition $\boldsymbol{c}_{K}\left(t_{0}\right)=\boldsymbol{e}_{K}^{0}\left(t_{0}\right)$ and consider the solution (21) obtained by expanding each $\boldsymbol{Q}_{K}(t)$ in expression (22). Noting $\boldsymbol{Q}_{K}\left(t_{0}\right) \boldsymbol{e}_{K}^{0}\left(t_{0}\right)$ is zero and that the $n$th order time derivatives of $\boldsymbol{Q}_{K}(t)$ scale with $\epsilon^{n}$ we have

$$
\boldsymbol{c}_{K}(t)=\boldsymbol{e}_{K}^{0}\left(t_{0}\right)+\sum_{n=1}^{\infty} \frac{\Delta t^{n+1}}{(n+1) !} \boldsymbol{Q}_{K}^{n-1}\left(t_{0}\right) \dot{\boldsymbol{Q}}_{K}\left(t_{0}\right) \boldsymbol{e}_{K}^{0}\left(t_{0}\right)+O\left(\epsilon^{2}\right)
$$

By differentiating $\boldsymbol{Q}_{K}(s) \boldsymbol{e}_{K}^{0}(s)=0$ with respect to $s$ and then setting $s=t_{0}$ we also have

$$
\dot{\boldsymbol{Q}}_{K}\left(t_{0}\right) \boldsymbol{e}_{K}^{0}\left(t_{0}\right)=-\boldsymbol{Q}_{K}\left(t_{0}\right) \dot{\boldsymbol{e}}_{K}^{0}\left(t_{0}\right)
$$

Let $\boldsymbol{e}_{K}^{0 P}(t)$ be the unit vector which is perpendicular to $\boldsymbol{e}_{K}^{0}(t)$ where $P$ denotes perpendicular. Because $\boldsymbol{e}_{K}^{0}(t)$ is defined to be a unit vector, we have

$$
\dot{\boldsymbol{e}}_{K}^{0}(t)=\frac{\epsilon}{\tau(t)} \boldsymbol{e}_{K}^{O P}(t)
$$


where $\tau(t)$ has an implicit $K$ dependence. Since the matrix $Q_{K}(t)$ changes on the growth timescale we also have that $\epsilon / \tau(t) \sim O\left(T_{\text {growth }}^{-1}\right)$ and hence $\tau(t) \sim O\left(T_{\text {dyn }}\right)$. We also have the kinematic relation

$$
\boldsymbol{e}_{K}^{1}(t)=\cos \psi(t) \boldsymbol{e}_{K}^{0 P}(t)+\sin \psi(t) \boldsymbol{e}_{K}^{0}(t)
$$

by projecting $\boldsymbol{e}_{K}^{1}(t)$ onto the $\boldsymbol{e}_{K}^{0}(t)$ and $\boldsymbol{e}_{K}^{0 P}(t)$ directions. Note that $\psi(t)$ has an implicit $K$ dependence and that, without loss of generality, $\psi(t) \in[0, \pi / 2)$. Hence

$$
\boldsymbol{Q}_{K}\left(t_{0}\right) \dot{\boldsymbol{e}}_{K}^{0}\left(t_{0}\right)=\frac{\lambda_{K}^{0}\left(t_{0}\right)}{\tau\left(t_{0}\right) \cos \psi\left(t_{0}\right)} \boldsymbol{e}_{K}^{1}(t)
$$

where $\lambda_{K}^{0}\left(t_{0}\right)<0$ is the negative eigenvalue of $\boldsymbol{Q}_{K}\left(t_{0}\right)$. By substituting this into Eq. (23) we have

$$
\begin{aligned}
\boldsymbol{c}_{K}(t)= & {\left[1+O\left(\epsilon^{2}\right)\right] \boldsymbol{e}_{K}^{0}\left(t_{0}\right) } \\
& +\left[\left.\frac{\epsilon \Delta t}{\tau\left(t_{0}\right) \cos \psi\left(t_{0}\right)}\left(\frac{e^{s}-(1+s)}{s}\right)\right|_{l s=\lambda_{K}^{0}\left(t_{0}\right) \Delta t}+O\left(\epsilon^{2}\right)\right] \boldsymbol{e}_{K}^{1}\left(t_{0}\right)
\end{aligned}
$$

which is not decaying.

To proceed we use Eqs. (18), (25) and the observation that

$$
\begin{aligned}
\int_{t_{0}}^{t_{0}+\Delta t} d s \lambda_{K}^{*}(s)= & \lambda_{K}^{*}\left(t_{0}+\frac{\Delta t}{2}\right) \Delta t \\
& +\ddot{\lambda}_{K}^{*}\left(t_{0}+\frac{\Delta t}{2}\right) \int_{t_{0}}^{t_{0}+\Delta t} d s\left(s-\left[t_{0}+\frac{\Delta t}{2}\right]\right)+\cdots \\
= & \lambda_{K}^{*}\left(t_{0}+\frac{\Delta t}{2}\right) \Delta t\left[1+O\left(\frac{\epsilon^{2} \Delta t^{2}}{T_{\text {dyn }}^{2}}\right)\right]
\end{aligned}
$$

where we have used the scaling relation $\ddot{\lambda}_{K}^{*} \sim O\left(\epsilon^{2} \lambda_{K}^{*} / T_{d y n}^{2}\right)$. Thus we have

$$
\frac{\left\|\boldsymbol{b}_{K}\left(t_{0}+\Delta t\right)\right\|}{\left\|\boldsymbol{b}_{K}\left(t_{0}\right)\right\|}=\exp \left[\lambda_{K}^{*}\left(t_{0}+\frac{\Delta t}{2}\right) \Delta t\right]\left[1+O\left(\epsilon^{2}\right)\right] \text {. }
$$

Thus we see, accurate to $O\left(\epsilon^{2}\right)$ corrections, that the growth rate with respect to the norm is given by

$$
\lambda_{K}^{G} \stackrel{\text { def }}{=} \lambda_{K}^{*}\left(t_{0}+\frac{\Delta t}{2}\right) .
$$

with $\lambda_{K}^{*}$ the largest real eigenvalue of $\boldsymbol{M}_{K}$. 


\section{On the breakdown of the perturbation expansion}

We briefly consider the nature of the $O\left(\epsilon^{2}\right)$ corrections. Noting $\tau\left(t_{0}\right) \sim T_{d y n}$ the $\left[1+O\left(\epsilon^{2}\right)\right]$ on the right hand side of Eq. (26) can be refined to

$$
\left[1+O\left(\frac{\epsilon^{2} \Delta t^{2}}{T_{d y n}^{2}}\right)+O\left(\frac{\epsilon^{2} \Delta t^{2}}{T_{d y n}^{2} \cos ^{2}\left(\psi\left(t_{0}\right)\right)}\right)+O\left(\frac{\epsilon^{2} \Delta t^{3} \lambda_{K}^{0}\left(t_{0}\right)}{T_{d y n}^{2} \cos ^{2}\left(\psi\left(t_{0}\right)\right)}\right)+O\left(\epsilon^{3}\right)\right] .
$$

The final $O\left(\epsilon^{2}\right)$ term arises from explicitly calculating the $O\left(\epsilon^{2}\right)$ contribution to the coefficient of $\mathbf{e}_{K}^{0}$ in Eq. (25), which is not presented for brevity.

It is evident that further comment is required for $\pi / 2-\psi\left(t_{0}\right) \ll 1$ as this will lead to a general invalidation of the perturbation method since we then have $\left|\cos \psi\left(t_{0}\right)\right| \ll$ 1. Under these circumstances, the eigenvectors of $\boldsymbol{Q}_{K}\left(t_{0}\right)$ and hence $\boldsymbol{M}_{K}\left(t_{0}\right)$, are extremely close to parallel. Even if the solution decays along one eigenmode direction, this can actually induce a large, transient, excursion in the phase plane. This may, by itself, be sufficient to induce an instability even in an autonomous system due to the influence of non-linear effects during such a large deviation. The fact this behaviour is both transient and requires contributions from the two eigenmodes and non-linearities entails this means of driving an instability is also neglected in the textbook Turing analysis (Murray 2002). It is therefore also not considered here especially given that requiring that the fastest growing mode corresponds to the case with nearly parallel eigenvectors necessitates an extreme fine tuning of parameters, which in itself is unreasonable to impose on a biological model. Henceforth we assume $\pi / 2-\psi\left(t_{0}\right) \ll 1$.

It is also clear that once $\Delta t / T_{d y n}$ starts becoming large relative to the inverse of the asymptotically small parameter, $\epsilon$, the structure of the asymptotic expansion breaks down. Nonetheless, the asymptotic structure is clearly valid up to $\Delta t / T_{d y n} \sim O(1)$. Thus, importantly, the allowed range of $\Delta t$ is not asymptotically small; instead it is sufficient to allow a perturbation to start growing, thus allowing a meaningful study of linearised stability.

\subsection{2 $\boldsymbol{M}_{K}(t)$ has complex conjugate eigenvalues}

The matrix $\boldsymbol{M}_{K}(t)$ has a complex eigenvector

$$
\boldsymbol{e}_{K}(t)=\boldsymbol{f}_{K}^{0}(t)+i \boldsymbol{f}_{K}^{1}(t)
$$

associated with the complex eigenvalue $\lambda(t)$, where $f_{K}^{0}(t), f_{K}^{1}(t)$ are real, distinct, vectors and thus a spanning set of the real plane. Without loss of generality we take $f_{K}^{0}(t)$ to be a unit vector and let $\Omega_{K} \stackrel{\text { def }}{=} \operatorname{Im}(\lambda(t))>0$. We can write any real vector function of time in the form

$$
c_{K}(t)=\alpha_{K}(t) \boldsymbol{f}_{K}^{0}(t)+\beta_{K}(t) \boldsymbol{f}_{K}^{1}(t)
$$

and define the norm

$$
\left\|\boldsymbol{c}_{K}(t)\right\|=\sqrt{\alpha_{K}^{2}(t)+\beta_{K}^{2}(t)} .
$$


With $\overline{\boldsymbol{e}}_{K}(t)$ denoting the conjugate of $\boldsymbol{e}_{K}(t)$ we have

$$
\begin{aligned}
\dot{\boldsymbol{e}}_{K}(t) & =\dot{\boldsymbol{f}}_{K}^{0}(t)+i \dot{\boldsymbol{f}}_{K}^{1}(t) \stackrel{\text { def }}{=} \epsilon A_{K} \boldsymbol{f}_{K}^{0}(t)+i \epsilon B_{K} \boldsymbol{f}_{K}^{1}(t) \\
& =\epsilon \frac{A_{K}+B_{K}}{2} \boldsymbol{e}_{K}(t)+\epsilon \frac{A_{K}-B_{K}}{2} \overline{\boldsymbol{e}}_{K}(t) \stackrel{\text { def }}{=} \epsilon \mathcal{A}_{K} \boldsymbol{e}_{K}(t)+\epsilon \mathcal{B}_{K} \overline{\boldsymbol{e}}_{K}(t)
\end{aligned}
$$

where $A_{K}, B_{K}, \mathcal{A}_{K}, \mathcal{B}_{K}$ are defined by the above relations and are time-dependent. The $\epsilon$ appears as the change in the eigenvector $\boldsymbol{e}_{K}(t)$ is on the timescale of growth. As long as the angle between $f_{K}^{0}$ and $f_{K}^{1}$ is significantly greater than $\epsilon$ and the vectors have a ratio of lengths, $l$, with $\epsilon \ll l \ll \epsilon^{-1}$, then $A_{K}(t)$ and $B_{K}(t)$ can be treated as $\mathrm{O}\left(\epsilon^{0}\right)$ terms for the purposes of asymptotic expansions. This is assumed below.

We also have that

$$
\boldsymbol{Q}_{K}(t) \boldsymbol{e}_{K}(t)=i \Omega_{K} \boldsymbol{e}_{K}(t), \quad \boldsymbol{Q}_{K}(t) \overline{\boldsymbol{e}}(t)=-i \Omega_{K} \overline{\boldsymbol{e}}(t)
$$

and hence, by differentiation,

$$
\begin{aligned}
\dot{\boldsymbol{Q}}_{K}(t) \boldsymbol{e}_{K}(t) & =-\left(\boldsymbol{Q}_{K}(t)-i \Omega_{K} \boldsymbol{I}\right) \dot{\boldsymbol{e}}_{K}(t)+i \dot{\Omega}_{K} \boldsymbol{e}_{K}(t) \\
& =i \epsilon \Omega_{K}\left[2 \mathcal{B}_{K} \overline{\boldsymbol{e}}_{K}(t)+\Phi_{K} \boldsymbol{e}_{K}(t)\right]
\end{aligned}
$$

where $\Phi_{K} \stackrel{\text { def }}{=} \dot{\Omega}_{K} /\left[\epsilon \Omega_{K}\right] \sim O\left(1 / T_{d y n}\right)$ as $\Omega_{K}$ changes on the growth timescale $T_{\text {growth }}=T_{d y n} / \epsilon$.

Thus for a general initial condition

$$
\boldsymbol{c}_{K}\left(t_{0}\right)=\alpha_{K} \boldsymbol{f}_{K}^{0}\left(t_{0}\right)+\beta_{K} \boldsymbol{f}_{K}^{1}\left(t_{0}\right)=\operatorname{Re}\left[\left(\alpha_{K}-i \beta_{K}\right) \boldsymbol{e}_{K}\left(t_{0}\right)\right]
$$

we have

$$
\begin{aligned}
& \boldsymbol{c}_{K}(t)=\operatorname{Re}\left[( \alpha _ { K } - i \beta _ { K } ) \left\{\exp \left[\boldsymbol{Q}_{K}\left(t_{0}\right) \Delta t\right] \boldsymbol{e}_{K}\left(t_{0}\right)+\sum_{n=1}^{\infty} \sum_{j=1}^{n} \int_{t_{0}}^{t} d t_{1} \int_{t_{0}}^{t_{1}} d t_{2} \ldots\right.\right. \\
& \left.\left.\times \int_{t_{0}}^{t_{n-2}} d t_{n-1} \int_{t_{0}}^{t_{n-1}} d t_{n}\left(t_{j}-t_{0}\right) \boldsymbol{Q}_{K}^{j-1}\left(t_{0}\right) \dot{\boldsymbol{Q}}_{K}\left(t_{0}\right) \boldsymbol{Q}_{K}^{n-j}\left(t_{0}\right) \boldsymbol{e}_{K}\left(t_{0}\right)\right\}\right]+O\left(\epsilon^{2}\right) \\
& =\operatorname{Re}\left[( \alpha _ { K } - i \beta _ { K } ) \left\{\exp \left[\boldsymbol{Q}_{K}\left(t_{0}\right) \Delta t\right] \boldsymbol{e}_{K}\left(t_{0}\right)+\epsilon \sum_{n=1}^{\infty} \sum_{j=1}^{n} \frac{\Delta t^{n+1}}{(n+1) !}[n-j+1]\right.\right. \\
& \left.\left.\times\left(i \Omega_{K}\right)^{n}\left[\Phi_{K} \boldsymbol{e}_{K}\left(t_{0}\right)+2 \mathcal{B}_{K}(-1)^{j-1} \overline{\boldsymbol{e}}_{K}\left(t_{0}\right)\right]\right\}\right]+O\left(\epsilon^{2}\right) \text {. } \\
& =\operatorname{Re}\left[\left(\alpha_{K}-i \beta_{K}\right) \times\left\{e^{i \Omega_{K} \Delta t}\left\{1+i \epsilon \frac{\Delta t^{2}}{2} \Phi_{K} \Omega_{K}\right\} \boldsymbol{e}_{K}\left(t_{0}\right)\right.\right. \\
& \left.\left.-\epsilon \mathcal{B}_{K}\left\{\frac{\sin \left(\Omega_{K} \Delta t\right)}{\Omega_{K}}-\Delta t e^{i \Omega_{K} \Delta t}\right\} \overline{\boldsymbol{e}}_{K}\left(t_{0}\right)\right\}\right]+O\left(\epsilon^{2}\right) \text {. }
\end{aligned}
$$


Calculating the norm reveals

$$
\begin{aligned}
\left\|\boldsymbol{c}_{K}(t)\right\|^{2}= & \left\|\boldsymbol{c}_{K}\left(t_{0}\right)\right\|^{2}\left\{1+2 \epsilon\left|\mathcal{B}_{K}\right| \Delta t\left[-\cos \left(2 \Omega_{K} \Delta t+\chi_{0}\right)\right.\right. \\
& \left.\left.+\frac{\sin \left(\Omega_{K} \Delta t\right)}{\Omega_{K} \Delta t} \cos \left(\Omega_{K} \Delta t+\chi_{0}\right)\right]\right\}+O\left(\epsilon^{2}\right),
\end{aligned}
$$

where

$$
\chi_{0}=\tan ^{-1}\left(\frac{2 \alpha_{K} \beta_{K} \operatorname{Re} \mathcal{B}_{K}+\left(\beta_{K}^{2}-\alpha_{K}^{2}\right) \operatorname{Im} \mathcal{B}_{K}}{\left(\beta_{K}^{2}-\alpha_{K}^{2}\right) \operatorname{Re} \mathcal{B}_{K}-2 \alpha_{K} \beta_{K} \operatorname{Im} \mathcal{B}_{K}}\right)=\arg \left(\left(\alpha_{K}-i \beta_{K}\right)^{2} \mathcal{B}_{K}\right)
$$

is a phase angle. We can rewrite the above norm in the form

$$
\begin{aligned}
\left\|\boldsymbol{c}_{K}(t)\right\|= & \left\|\boldsymbol{c}_{K}\left(t_{0}\right)\right\|\left\{1+\epsilon\left|\mathcal{B}_{K}\right| \Delta t\left[1-2 \cos \left(\Omega_{K} \Delta t\right) \frac{\sin \left(\Omega_{K} \Delta t\right)}{\Omega_{K} \Delta t}\right.\right. \\
& \left.\left.+\frac{\sin ^{2}\left(\Omega_{K} \Delta t\right)}{\left[\Omega_{K} \Delta t\right]^{2}}\right]^{1 / 2} \cos \left(\Omega_{K} \Delta t+\chi_{1}\right)\right\}+O\left(\epsilon^{2}\right),
\end{aligned}
$$

where

$$
\chi_{1}=\chi_{0}+\tan ^{-1}\left(\frac{\sin \left(\Omega_{K} \Delta t\right)}{\cos \left(\Omega_{K} \Delta t\right)-\frac{\sin \left(\Omega_{K} \Delta t\right)}{\Omega_{K} \Delta t}}\right)
$$

Thus we have an oscillating solution, the amplitude of which is constant at leading order but not at higher orders. One can immediately deduce that, accurate to $O\left(\epsilon^{2}\right)$

$$
\frac{\left\|\boldsymbol{b}_{K}\left(t_{0}+\Delta t\right)\right\|}{\left\|\boldsymbol{b}_{K}\left(t_{0}\right)\right\|}=\exp \left[\Delta t\left\{\lambda_{K}^{*}\left(t_{0}+\frac{\Delta t}{2}\right)+\epsilon\left|\mathcal{B}_{K}\left(t_{0}\right)\right| \mathcal{C}\left(\Omega_{K} \Delta t, \chi_{1}\right)\right\}\right]
$$

with

$\mathcal{C}\left(\Omega_{K} \Delta t, \chi_{1}\right) \stackrel{\text { def }}{=}\left[1-2 \cos \left(\Omega_{K} \Delta t\right) \frac{\sin \left(\Omega_{K} \Delta t\right)}{\Omega_{K} \Delta t}+\frac{\sin ^{2}\left(\Omega_{K} \Delta t\right)}{\left[\Omega_{K} \Delta t\right]^{2}}\right]^{1 / 2} \cos \left(\Omega_{K} \Delta t+\chi_{1}\right)$

note that $\mathcal{B}_{K}$ is evaluated at time $t_{0}$. 
The important result for investigating stability is that an $O\left(\epsilon^{2}\right)$ accurate estimate of the growth rate between time $t_{0}$ and time $t$ is given by

$$
\begin{aligned}
\lambda_{K}^{G} \stackrel{\text { def }}{=} & \lambda_{K}^{*}\left(t_{0}+\frac{\Delta t}{2}\right)+\epsilon\left\{| \mathcal { B } _ { K } ( t _ { 0 } ) | \left[1-2 \cos \left(\Omega_{K} \Delta t\right) \frac{\sin \left(\Omega_{K} \Delta t\right)}{\Omega_{K} \Delta t}\right.\right. \\
+ & \left.\left.\frac{\sin ^{2}\left(\Omega_{K} \Delta t\right)}{\Omega_{K}^{2} \Delta t^{2}}\right]^{1 / 2} \cos \left(\Omega_{K} \Delta t+\chi_{1}\right)\right\}
\end{aligned}
$$

with $\lambda_{K}^{*}$ denoting the real part of the eigenvalues of $\boldsymbol{M}_{K}$. Once more we assume that the perturbation does not break down due to a near degeneracy of the eigenvectors of $M_{K}$ and that $\Delta t \sim T_{d y n}$.

\subsection{3 $\boldsymbol{M}_{K}(t)$ has real, repeated eigenvalues}

This scenario requires a mathematically precise parameter fine tuning, and thus is unlikely to occur in most models. The matrix $\boldsymbol{Q}_{K}(t)$ in Eq. (19) now has a repeated zero eigenvalue, one eigenvector only, and has zero square. Expanding Eqs. (21) and (22) as above, but now for general initial conditions and utilising $Q_{K}^{2}(t)=0$ we have

$$
\begin{aligned}
\boldsymbol{c}_{K}(t)= & {\left[\boldsymbol{I}+\Delta t \boldsymbol{Q}_{K}\left(t_{0}\right)+\frac{(\Delta t)^{2}}{2} \dot{\boldsymbol{Q}}_{K}\left(t_{0}\right)+\frac{(\Delta t)^{3}}{6} \boldsymbol{Q}_{K}\left(t_{0}\right) \dot{\boldsymbol{Q}}_{K}\left(t_{0}\right)\right] \boldsymbol{c}_{K}\left(t_{0}\right) } \\
& +\left[\frac{(\Delta t)^{3}}{3} \dot{\boldsymbol{Q}}_{K}\left(t_{0}\right) \boldsymbol{Q}_{K}\left(t_{0}\right)+\frac{(\Delta t)^{4}}{12} \boldsymbol{Q}_{K}\left(t_{0}\right) \dot{\boldsymbol{Q}}_{K}\left(t_{0}\right) \boldsymbol{Q}_{K}\left(t_{0}\right)\right] \boldsymbol{c}_{K}\left(t_{0}\right)+O\left(\epsilon^{2}\right) .
\end{aligned}
$$

The nilpotency of $\boldsymbol{Q}_{K}(t)$ truncates the series, which entails polynomial behaviour in time; in fact the final $O\left(\Delta t^{4}\right)$ term above will not contribute due to nilpotency, though this requires explicit calculation. Thus, the governing dynamics of $\boldsymbol{b}_{K}(t)$ is not simply exponential growth (or decay) at a rate determined, even approximately, by an eigenvalue of $\boldsymbol{M}_{K}(t)$. In contrast to the non-autonomous case, we have nonlinear polynomial terms at $O(\epsilon)$ in an asymptotic expansion. Thus a study of this case does not fit naturally into the current study or the standard analysis of the nonautonomous case. Furthermore, eigenvalue calculations in the presence of repeated roots of the characteristic equation can be exquisitely sensitive to matrix perturbations Golub and Van Loan (1996). Consequently, a special study would have to be conducted for repeated eigenvalues. However, requiring the fastest growing mode corresponding to the case of repeated eigenvalues demands a mathematically precise fixing of parameter values, which is inappropriate for biological models. Thus we do not consider this special case below.

Summary Reconsider Eqs. (14) to (17), plus Eqs. (27) and (29). We have $O\left(\epsilon^{2}\right)$ accurate expressions for the growth rate of $\boldsymbol{b}_{K}(t)$ and hence the growth rate of the perturbation $\boldsymbol{a}_{K}(t)$, at least when degenerate or near-degenerate cases, such as those 
described in Sect. (3.3.3), above are excluded. Thus a study of stability reduces to determining whether these growth rates are positive or negative.

\subsection{Turing diffusively-driven instability conditions on growing domains}

Given we now have an understanding of how the non-autonomous solutions behave, we can start to consider the Turing instability in detail. We consider a perturbation at time $t_{0}$, which can be distinct from the initial time, and how it has evolved by time $t=t_{0}+\Delta t>t_{0}$ with

$$
\frac{\Delta t}{T_{d y n}} \sim O\left(\epsilon^{0}\right)
$$

where $\epsilon$ is defined in Sect. (2.1.2). It will be useful to define

$$
t_{1} \stackrel{\text { def }}{=} \frac{t_{0}+t}{2}
$$

for use below. We first of all summarise our caveats. For any given mode we assume the matrix $\boldsymbol{M}_{K}$ does not have repeated eigenvalues, or a switch between real and complex eigenvalues for the interval $\left[t_{0}, t\right]$. If the eigenvalues are real, we also assume that the eigenvectors are not close to parallel, as defined by $\pi / 2-\psi \sim O(1)$ in Eq. (24). If the matrix $\boldsymbol{M}_{K}$ has complex eigenvalues, we analogously assume that the real and imaginary parts of the complex eigenvector are not nearly parallel (or anti-parallel), and that their ratio of lengthscales can be considered to be the order $O\left(\epsilon^{0}\right)$ in an asymptotic expansion. We also define

$$
h_{*}=h\left(t_{1}\right)
$$

when the eigenvalues of $\boldsymbol{M}_{K}\left(t_{1}\right)$ are real and

$h_{*}=h\left(t_{1}\right)-\left.\epsilon\left\{\left|\mathcal{B}_{K}\left(t_{0}\right)\right|\left[1-2 \cos (s) \frac{\sin (s)}{s}+\frac{\sin ^{2}(s)}{s^{2}}\right]^{1 / 2} \cos \left(s+\chi_{1}\right)\right\}\right|_{s=\Omega_{K} \Delta t}$

when the eigenvalues of $\boldsymbol{M}_{K}\left(t_{1}\right)$ are complex, with $\Omega_{K}$ the imaginary part of the complex eigenvalue and with $\mathcal{B}_{K}$ defined via Eq. (28). The latter arises from the timedependent rotation of the complex eigenvector of $\boldsymbol{M}_{K}\left(t_{0}\right)$. Given the above assumptions we also have $\left|\mathcal{B}_{K}\right| \sim O\left(\epsilon^{0} / T_{d y n}\right)$.

We are now in a position to state the main result of our paper:

The necessary conditions for a Turing diffusively-driven instability (accurate to first order in $\epsilon \ll 1)$ when considering the system at time $t$ due to a perturbation at time $t_{0}<t$ in the presence of spatially linear and isotropic growth as in system (8), are given by 


$$
\begin{aligned}
\gamma\left(f_{u}+g_{v}\right)-2 h_{*} & <0, \\
\gamma^{2}\left(f_{u} g_{v}-f_{v} g_{u}\right)-h_{*} \gamma\left(f_{u}+g_{v}\right) & >0, \\
h_{*}(1+d)-\gamma\left(d f_{u}+g_{v}\right) & <0, \\
{\left[h_{*}(1+d)-\gamma\left(d f_{u}+g_{v}\right)\right]^{2}-4 d\left[\gamma^{2}\left(f_{u} g_{v}-f_{v} g_{u}\right)-\gamma h_{*}\left(f_{u}+g_{v}\right)\right] } & >0 .
\end{aligned}
$$

In the above, the subscripts $u, v$ denote partial differentiation, with the Jacobian components $f_{u}, f_{v}, g_{u}$ and $g_{v}$ evaluated in terms of $\boldsymbol{u}_{S}\left(t_{1}\right)$. Strictly, we have $h_{*} \sim O(\epsilon)$, due to the fact that the growth timescale is much longer than any other timescale so that one may self-consistently neglect $O\left(h_{*}^{2}\right)$ corrections in the above.

\section{Derivation}

Let $\lambda_{K}^{*}\left(t_{1}\right)$ denote the largest real part of any eigenvalue of $\boldsymbol{M}_{K}\left(t_{1}\right)$. Given our assumptions and observations on the behaviour of the non-autonomous solutions, we have that the mode $\boldsymbol{w}_{K}\left(\xi, t_{1}\right)$, will grow during the interval $\left[t_{0}, t\right]$ if

$$
\mu_{K}^{*} \stackrel{\text { def }}{=} \lambda_{K}^{*}\left(t_{1}\right)-h_{*}>0
$$

and decay if

$$
\mu_{K}^{*} \stackrel{\text { def }}{=} \lambda_{K}^{*}\left(t_{1}\right)-h_{*}<0
$$

with $t_{1}=\left(t_{0}+t\right) / 2$, as above. By considering conditions for stability in the presence of spatially homogeneous perturbations and instability for spatially varying perturbations, we can deduce the above conditions for a diffusively-driven instability.

To proceed, we need an expression for $\lambda_{K}\left(t_{1}\right)$, which denotes an eigenvalue of $\boldsymbol{M}_{K}\left(t_{1}\right)$ with largest real part. This is the root of the quadratic equation

$$
\operatorname{det}\left[\boldsymbol{I} \lambda_{K}^{*}\left(t_{1}\right)+\frac{K^{2}}{\varphi^{2}\left(t_{1}\right)} \boldsymbol{D}-\left.\gamma \boldsymbol{J}_{\boldsymbol{F}}\right|_{\boldsymbol{u}_{S}\left(t_{1}\right)}\right]=0
$$

with largest real part. Thus $\lambda_{K}\left(t_{1}\right)$ satisfies the dispersion relation

$$
\lambda_{K}^{2}\left(t_{1}\right)+b\left(K_{*}^{2}\right) \lambda_{K}\left(t_{1}\right)+c\left(K_{*}^{2}\right)=0
$$

where

$$
\begin{gathered}
K_{*}^{2} \stackrel{\text { def }}{=} K^{2} / \varphi^{2}\left(t_{1}\right) \\
b\left(K_{*}^{2}\right)=K_{*}^{2}(1+d)-\gamma\left(f_{u}+g_{v}\right), \\
c\left(K_{*}^{2}\right)=d K_{*}^{4}-\gamma\left(d f_{u}+g_{v}\right) K_{*}^{2}+\gamma^{2}\left(f_{u} g_{v}-f_{v} g_{u}\right),
\end{gathered}
$$

with $u, v, f(u, v), g(u, v)$ the scalar variables and kinetic functions in Eq. (1). The partial derivatives are evaluated in terms of $\boldsymbol{u}_{S}\left(t_{1}\right)$ the solution to Eq. (11) at time $t_{1}$. 
Hence

$$
\mu_{K} \stackrel{\text { def }}{=} \lambda_{K}\left(t_{1}\right)-h_{*}
$$

satisfies

$$
\mu_{K}^{2}+\left(2 h_{*}+b\left(K_{*}^{2}\right)\right) \mu_{K}+\left(c\left(K_{*}^{2}\right)+b\left(K_{*}^{2}\right) h_{*}+O\left(h_{*}^{2}\right)\right)=0
$$

where $b\left(K_{*}^{2}\right)$ and $c\left(K_{*}^{2}\right)$ are given in Eqs. (37) and (38) respectively. Defining

$$
B_{h_{*}}\left(K_{*}^{2}\right)=2 h_{*}+b\left(K_{*}^{2}\right), \quad \text { and } \quad C_{h_{*}}\left(K_{*}^{2}\right)=c\left(K_{*}^{2}\right)+b\left(K_{*}^{2}\right) h_{*}+O\left(h_{*}^{2}\right)
$$

we obtain the characteristic equation

$$
\mu_{K}^{2}+B_{h_{*}}\left(K_{*}^{2}\right) \mu_{K}+C_{h_{*}}\left(K_{*}^{2}\right)=0
$$

Thus

$$
2 \mu_{K}=-B_{h_{*}}\left(K_{*}^{2}\right) \pm \sqrt{B_{h_{*}}^{2}\left(K_{*}^{2}\right)-4 C_{h_{*}}\left(K_{*}^{2}\right)}
$$

- With $K_{*}=0$ we have the absence of diffusion and thus spatial homogeneity. Requiring that for $\boldsymbol{u}_{S}\left(t_{1}\right)$ to be stable to the $K_{*}=0$, spatially homogeneous, mode entails

$$
\mu_{K}^{*}=\operatorname{Re}\left[\mu_{K}\right]=\operatorname{Re}\left[-B_{h_{*}}(0) \pm \sqrt{B_{h_{*}}^{2}(0)-4 C_{h_{*}}(0)}\right]<0 .
$$

This is guaranteed provided $B_{h_{*}}(0)>0, C_{h_{*}}(0)>0$ which respectively yield Eqs. (30) and (31) given by

$$
\begin{gathered}
2 h_{*}-\gamma\left(f_{u}+g_{v}\right)>0, \\
O\left(h_{*}^{2}\right)-h_{*} \gamma\left(f_{u}+g_{v}\right)+\gamma^{2}\left(f_{u} g_{v}-f_{v} g_{u}\right)>0,
\end{gathered}
$$

Thus we have Eqs. (30) and (31), which enforce the requirement that the homogeneous steady state is stable with respect to spatially homogeneous perturbations. Also note that from inequality (43), one can readily deduce that the left hand side of the condition in (44) represents an increasing function of $h_{*}$.

- In the presence of diffusion $\left(K_{*}^{2}>0\right)$, we have

$$
B_{h_{*}}\left(K_{*}^{2}\right)=2 h_{*}+b\left(K_{*}^{2}\right)=K_{*}^{2}(1+d)+B_{h_{*}}(0)>0
$$

since $B_{h_{*}}(0)>0$. For $\boldsymbol{u}_{S}\left(t_{1}\right)$ to become unstable, we require that

$$
\mu_{K}^{*}=\operatorname{Re}\left[\mu_{K}\right]>0 \text { for some } K_{*}^{2} \text { non-zero, }
$$


thereby requiring that $C_{h_{*}}\left(K_{*}^{2}\right)<0$ for some $K_{*}^{2}$ non-zero. By definition of $C_{h_{*}}\left(K_{*}^{2}\right)$ we can further re-arrange to obtain a quadratic polynomial in $K_{*}^{2}$ of the form

$$
C_{h_{*}}\left(K_{*}^{2}\right)=P_{2} K_{*}^{4}+P_{1} K_{*}^{2}+C_{h_{*}}(0)
$$

where

$$
\begin{aligned}
P_{2} & =d>0, \\
P_{1} & =h_{*}(1+d)-\gamma\left(d f_{u}+g_{v}\right), \\
C_{h_{*}}(0) & =c(0)+b(0) h_{*}+O\left(h_{*}^{2}\right) \\
& =\gamma^{2}\left(f_{u} g_{v}-f_{v} g_{u}\right)-\gamma h_{*}\left(f_{u}+g_{v}\right)+O\left(h_{*}^{2}\right) .
\end{aligned}
$$

By condition (44), $C_{h_{*}}(0)>0$, and therefore we will require that $P_{1}<0$ in order for $C_{h_{*}}\left(K_{*}^{2}\right)<0$ for some non-zero $K_{*}^{2}$. Hence one of the conditions (32) for diffusively-driven instability is given by

$$
h_{*}(1+d)-\gamma\left(d f_{u}+g_{v}\right)<0 .
$$

For diffusively-driven instability to occur, we also require that there exists real $K_{ \pm}^{2}$ such that $C_{h_{*}}\left(K_{ \pm}^{2}\right)=0$ and these can be easily shown to be given by

$$
K_{ \pm}^{2}=\frac{-P_{1} \pm \sqrt{P_{1}^{2}-4 C_{h_{*}}(0) d}}{2 d} .
$$

Thus, requiring $C_{h_{*}}\left(K_{*}^{2}\right)<0$ entails $P_{1}^{2}-4 C_{h_{*}}(0) d>0$, thereby yielding the last condition (33) for diffusively-driven instability:

$$
\begin{aligned}
& {\left[h_{*}(1+d)-\gamma\left(d f_{u}+g_{v}\right)\right]^{2}} \\
& \quad-4 d\left[\gamma^{2}\left(f_{u} g_{v}-f_{v} g_{u}\right)-\gamma h_{*}\left(f_{u}+g_{v}\right)+O\left(h_{*}^{2}\right)\right]>0 .
\end{aligned}
$$

Now neglecting $O\left(h_{*}^{2}\right)$ results in the inequalities (30)-(33). In addition to these inequalities, a Turing diffusively-driven instability requires that there exists at least one wavenumber such that $K_{*}^{2}$ is contained in the interval

$$
K_{*}^{2} \in\left(K_{-}^{2}, K_{+}^{2}\right)
$$

where $K_{ \pm}^{2}$ are given by Eq. (49).

Note that the above conditions generalise the classic results for the case of fixed domains (Turing 1952; Edelstein-Keshet 1988; Murray 2002). In addition, the inequalities (30)-(33) define a time-dependent domain in parameter space, generalising the Turing space. On growing domains, this generalised Turing parameter space is contingent on domain growth. 


\subsubsection{Similarities and differences on comparison with the autonomous case}

We are now in a position to see which properties of the autonomous Turing diffusivelydriven instability conditions are inherited in the presence of growth perturbations and, more importantly, we will highlight any properties that are not.

1. As in the autonomous system, note that if $L$ is a characteristic lengthscale of the domain, then by applying a scaling argument to Eq. (13), permissible wavenumbers are such that $K^{2} L^{2}$ is constant as $L$ varies. For example on a onedimensional domain with zero flux conditions one has $K^{2} L^{2} / \pi^{2}$ is a square integer. Thus on a sufficiently small domain, all permissible modes are such that $K_{*}^{2}=K^{2} / \varphi^{2}\left(t_{1}\right)>K_{+}^{2}$, where $K_{+}^{2}$ is given by Eq. (49) and is $L$-independent. Hence the system is stable on a sufficiently small domain but as $L$ increases, a diffusively-driven instability will spontaneously emerge if inequalities (30)-(33) are satisfied.

2. Similarly to the autonomous case, a diffusively-driven instability cannot occur for equal diffusion coefficients. Setting $d=1$ in inequalities (30) and (32) gives

$$
\gamma\left(f_{u}+g_{v}\right)<2 h_{*}<\gamma\left(f_{u}+g_{v}\right)
$$

which is a contradiction.

3. Similarly to the autonomous case, a diffusively-driven instability cannot occur via a Hopf bifurcation. The instability final condition (33) is equivalent to $C_{h_{*}}\left(K_{*}^{2}\right)<$ 0 ; combined with Eq. (42) this enforces $\operatorname{Im}\left(\mu_{K}\right)=0$ and hence no instability is oscillatory in nature. Thus when an instability does occur we have that $\boldsymbol{M}_{K}$ has real eigenvalues (within the range $\left[t_{0}, t\right]$ under consideration), and hence we have $h_{*}=h\left(t_{1}\right)$ in the instability conditions (30)-(33).

4. In contrast to the autonomous case, if we have an activator and an inhibitor, we do not require short-range activation, long-range inhibition which is the standard mechanism for a diffusively-driven instability. For example, suppose without loss of generality that $u$ is the activator in Eq. (1) and $v$ is the inhibitor. Thus $f_{u}>0$ and $g_{v}<0$. Again from inequalities (30) and (32) we require

$$
0>\gamma\left(g_{v}+f_{u}\right)-2 h_{*}>(d-1)\left(h_{*}-\gamma f_{u}\right),
$$

where $\gamma>0$. In the absence of growth, $h_{*}$ is zero and we immediately have $0>(1-d) f_{u}$ and hence $1-d$ is negative. Thus, the inhibitor has to diffuse faster than the activator in the absence of growth for an instability to be possible. However for $h_{*} \neq 0$, we instead simply have $0>(d-1)\left(h_{*}-\gamma f_{u}\right)$ Thus we can have $0<\gamma f_{u}<h_{*}$ implying $d<1$. Hence, in the presence of growth, short-range inhibition, long-range activation can also generate a diffusively-driven instability.

5. In contrast to the autonomous case an activator and inhibitor interaction is not required to generate an instability; for example, two activators can satisfy the instability constraints. Again suppose that $u$ is the activator in Eq. (1) so that 
$f_{u}>0$. Using inequality (30) we find

$$
g_{v}<\left(\frac{2 h_{*}}{\gamma}-f_{u}\right)<\frac{2 h_{*}}{\gamma},
$$

where $\gamma>0$. Thus, for $h_{*}=0$, as with no growth, we have $g_{v}<0$, implying that $v$ must be an inhibitor. In contrast for $h_{*}>0$ we clearly are not required to have $g_{v}<0$ and hence the biochemical corresponding to the concentration $v$ can also be an activator without making a diffusively-driven instability impossible. Therefore an activator-activator mechanism could give rise to Turing patterns only in the presence of domain growth. These will be termed domain-growth-induced Turing patterns.

Even though we have not been able to consider cases of the most extreme eigenvalue sensitivity to matrix perturbations, such as the presence of repeated roots, the latter examples highlight that the instability conditions (30)-(33) are fundamentally different from the conditions in the autonomous case. Thus, while growth does lead to enhanced pattern robustness, by forcing pattern selection as the system grows, growth does in fact enhance instability in that more general choices of kinetics will exhibit a diffusively-driven instability. Below, we illustrate how the Turing space changes with growth and illustrate how a system which has $f_{u} g_{v}>0$, and thus cannot undergo a diffusively-driven instability in the absence of growth, undergoes peak formation with slow growth.

\section{Numerical experiments}

To illustrate our theoretical findings, we firstly briefly introduce slow, isotropic growth for linear, exponential and logistic evolution of the domain, calculating growth functions such as $h(t)$ required for the subsequent explorations. Then two examples are considered in detail. The first is an activator-depleted model known to satisfy the cross/pure kinetics conditions (Gierer and Meinhardt 1972; Prigogine and Lefever 1968; Schnakenberg 1979). The second example consists of reaction kinetics which do not satisfy any of the cross/pure kinetics conditions. Our aims are to show that the Turing space can be substantially altered by even slow growth and, further, that the effects of incorporating domain growth does not necessarily restrict one to consider only a short range activator-long range inhibition mechanism. This is in distinct contrast to pattern formation on fixed domains.

\subsection{Examples: uniform, isotropic growth}

Let us assume that the domain growth is spatially linear and, in higher spatial dimensions, isotropic. Without any loss of generality, let us assume that $t_{0}=0$. Let $\boldsymbol{x}(t)=$ $\xi \varphi(t)$ where $\varphi(t)>0$ is the domain growth function satisfying $\varphi(0)=1$. We can 
Table 1 Table illustrating the function $h(t)$ for linear, exponential and logistic growth functions

\begin{tabular}{|c|c|c|c|}
\hline Type of growth & Growth function $\varphi(t)$ & $h(t)=m S(t)$ & $q(t)=e^{-\int_{t_{0}}^{t} h(\tau) d \tau}$ \\
\hline Linear & $\varphi(t)=r t+1$ & $h(t)=\frac{m r}{r t+1}$ & $q(t)=\left(\frac{1}{r t+1}\right)^{m}$ \\
\hline Exponential & $\varphi(t)=e^{r t}$ & $h(t)=m r$ & $q(t)=e^{-m r t}$ \\
\hline Logistic & $\varphi(t)=\frac{\kappa A e^{\kappa r t}}{1+A e^{\kappa r t}}, A=\frac{1}{\kappa-1}$ & $h(t)=m r(\kappa-\varphi(t))$ & $q(t)=\left(\frac{e^{-\kappa r t}+A}{1+A}\right)^{m}$ \\
\hline
\end{tabular}

Note that $q(t)$ is as defined and used in Sect. 3. $\kappa$ is the carrying capacity (final domain size) corresponding to the logistic growth function

compute the domain (mesh) velocity as

$$
\left.\boldsymbol{a}(\boldsymbol{x}, t) \stackrel{\text { def }}{=} \frac{\partial \boldsymbol{x}}{\partial t}\right|_{\boldsymbol{\xi}}=\boldsymbol{\xi} \dot{\varphi}(t)=\frac{\dot{\varphi}(t)}{\varphi(t)} \boldsymbol{x}
$$

and hence it can be easily shown that

$$
h(t):=m S(t)=m \frac{\dot{\varphi}(t)}{\varphi(t)} \sim O\left(\frac{m \epsilon}{T_{d y n}}\right),
$$

where $m$ defines the number of spatial dimensions. In Table 1 we show the corresponding $h(t)$ for linear, exponential and logistic growth functions, with $\kappa \neq 1$. The definition of $A$ in this table ensures that $\varphi(0)=1$.

\subsection{Activator-depleted model: an illustrative example}

For illustrative purposes let us consider the activator-depleted substrate model (Gierer and Meinhardt 1972; Prigogine and Lefever 1968; Schnakenberg 1979) also known as the Brusselator model given by

$$
\begin{aligned}
& f(u, v)=a-u+u^{2} v \\
& g(u, v)=b-u^{2} v
\end{aligned}
$$

where $a$ and $b$ are positive parameters. In all our simulations we fix $d=10, \gamma=1$, $m=2$ and vary $a$ and $b$ as illustrated below.

\subsubsection{Exponential growth}

The Turing conditions for the exponential growth are independent of time $t_{1}$ since $h_{*}=h\left(t_{1}\right)=h(t):=m r$. For this special case, if $r$ is fixed, the Turing space obtained does not change in time. This is not the case for the linear and logistic growth. Since the conditions are time-independent, we illustrate different scenarios for different values of $r$. 


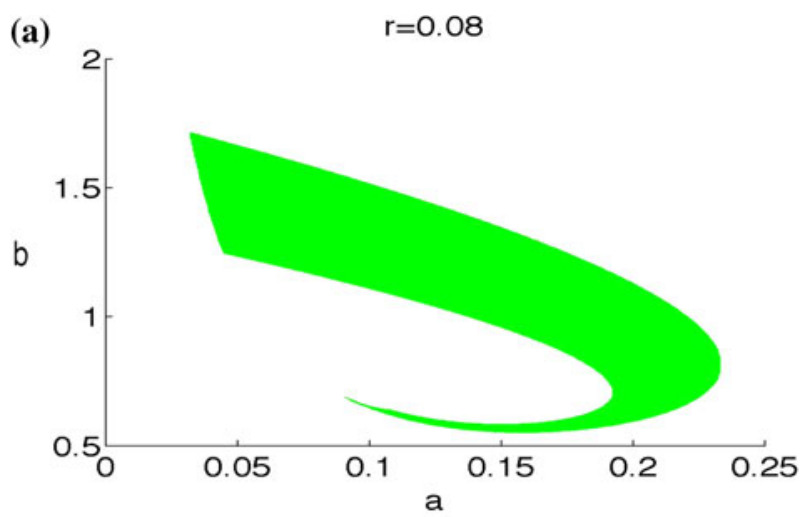

(b) $\quad r=0.16$
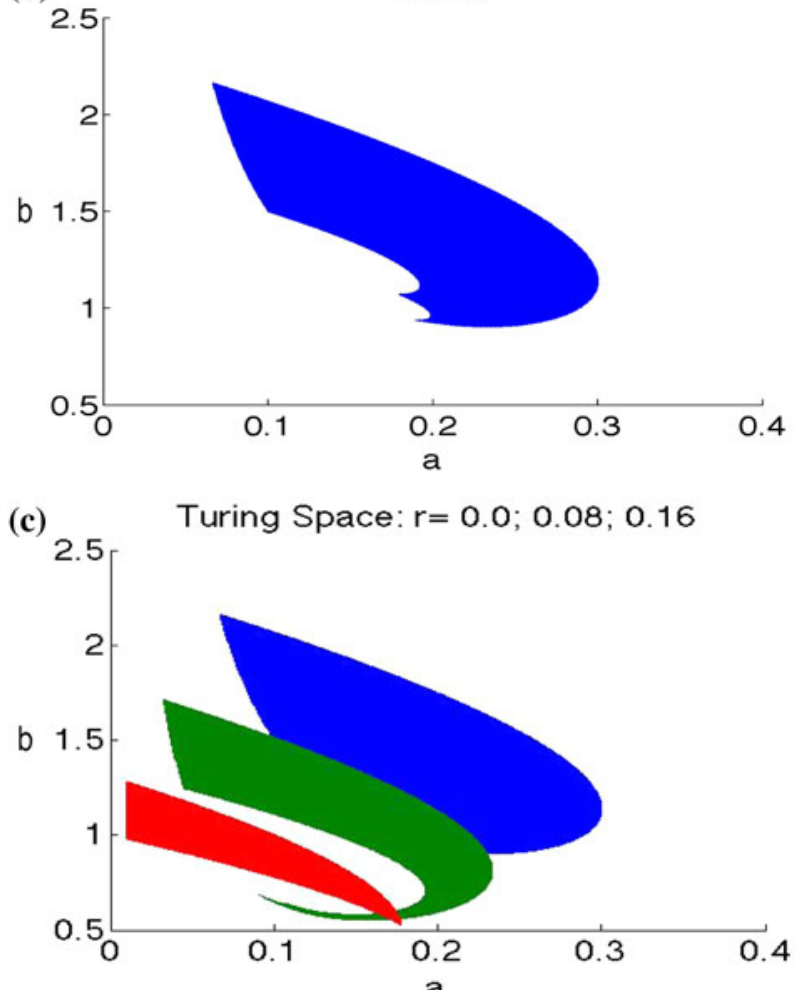

Fig. 1 Turing spaces for different exponential growth rates: (a) $r=0.08$, (b) $r=0.16$ and (c) the superimposition of spaces for $r=0$ (leftmost), $r=0.08$ (central) and $r=0.16$ (rightmost)

Figure 1 illustrates scenarios for different values of $r$. In order to highlight specific features of each space, we show Turing spaces for $r=0.08$ and 0.16 separately as shown in (a) and (b) and then superimpose them together with the space obtained in the absence of domain growth (c). The lower space (red) in (c) corresponds to 
the case of a fixed domain, i.e. $r=0$. The middle (green) and upper (blue) spaces correspond to the exponential growth rates $r=0.08$ and $r=0.16$ respectively. If $r$ is chosen too small $(r \ll 1$ ), say $r=0.002$ (Crampin et al. 1999; Madzvamuse et al. 2003; Madzvamuse 2005; Madzvamuse and Maini 2007; Madzvamuse 2008), then there are only negligible differences between the Turing spaces at $r=0$ and $r=0.002$ (results not shown). It is observed that for $r=0.08$ there is a small region where the space on fixed domain overlaps with that on an exponential growth. For relatively large values of $r$ there is no overlap between the Turing spaces on fixed and growing domains (for example spaces for $r=0$ and $r=0.16$ ). The fact that in general there is no overlap between the zero-growth rate Turing space and those on growing domains, allows us to conclude that, in general, diffusively-driven instability in the absence of growth need not imply diffusively-driven instability in the presence of growth.

\subsubsection{Linear and logistic growth functions}

For the linear and logistic growth functions we compute the time-dependent solutions $\left(u_{S}(t), v_{S}(t)\right)$ which satisfy the differential equations

$$
\begin{aligned}
& \frac{d u_{S}}{d t}=\gamma a-(\gamma+h(t)) u_{S}+\gamma u_{S}^{2} v_{S}, \\
& \frac{d v_{S}}{d t}=\gamma b-h(t) v_{S}-\gamma u_{S}^{2} v_{S}
\end{aligned}
$$

where $h(t)=m S(t)$ and given initial values at time $t_{0}=0$. From the linear growth function, $h(t)=\frac{m r}{r t+1}$ and similarly for the logistic growth function, $h(t)=m r(\kappa-$ $\varphi(t))$ where $\varphi(t)=\frac{A \kappa e^{\kappa r t}}{1+A e^{\kappa r t}}$, with $A=\frac{1}{\kappa-1}$. Let us take $r=0.1(\ll 1), m=$ $2.0, \kappa=2$ and $A=1$. It is clear from the definitions of the linear and logistic growth functions that as $t \longrightarrow \infty, h(t) \longrightarrow 0$. Therefore in the same large time limit, we also have that $\left(u_{S}(t), v_{S}(t)\right)$ tends to $\left(u_{S}, v_{S}\right)=\left(a+b, \frac{b}{(a+b)^{2}}\right)$ via damped oscillations. Note that $\left(u_{S}, v_{S}\right)$ represents the steady state in the absence of domain growth and is a global attractor for the system of non-autonomous ordinary differential equations. Fixing the model parameters $a=0.1, b=0.9$ and $\gamma=1$ and we plot the phase-diagrams corresponding to Eqs. (53) and (54) as illustrated in Fig. 2. In both the linear and logistic growth cases, a stable spiral point exists.

Next, we compute the Turing inequalities given linear and logistic growth functions respectively. The system of non-autonomous differential Eqs. (53)-(54) must be solved for every point $(a, b)$ in the plane at any given time $t$ thereby giving rise to the time-dependent Turing spaces. For every point $(a, b)$ in the plane, we take the initial conditions $u(0)=a+b$ and $v(0)=\frac{b}{(a+b)^{2}}$ and these correspond to the uniform steady state of the reaction-diffusion system in the absence of domain growth. Solving Eqs. (53) and (54) allows us to determine $h_{*}$ for varying $t_{1}$ given a growth rate of $r=0.1$, and we list these in Table 2. Note that for the chosen 
Fig. 2 Phase diagrams corresponding to the non-autonomous system of ordinary differential Eqs. (53)-(54) with (a) exponential, (b) linear and (c) logistic growth functions. A stable limit cycle exists for the exponential growth while a stable spiral point exists for the linear and logistic growth profiles. Here we have taken parameter values $a=0.1$, $b=0.9$ and $\gamma=1$ and the growth parameter values: $r=0.1, m=2, \kappa=2$ and A $=1$

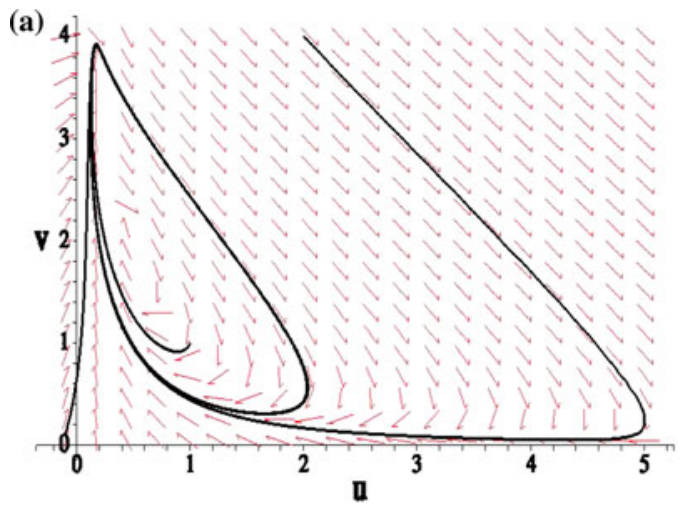

(b)
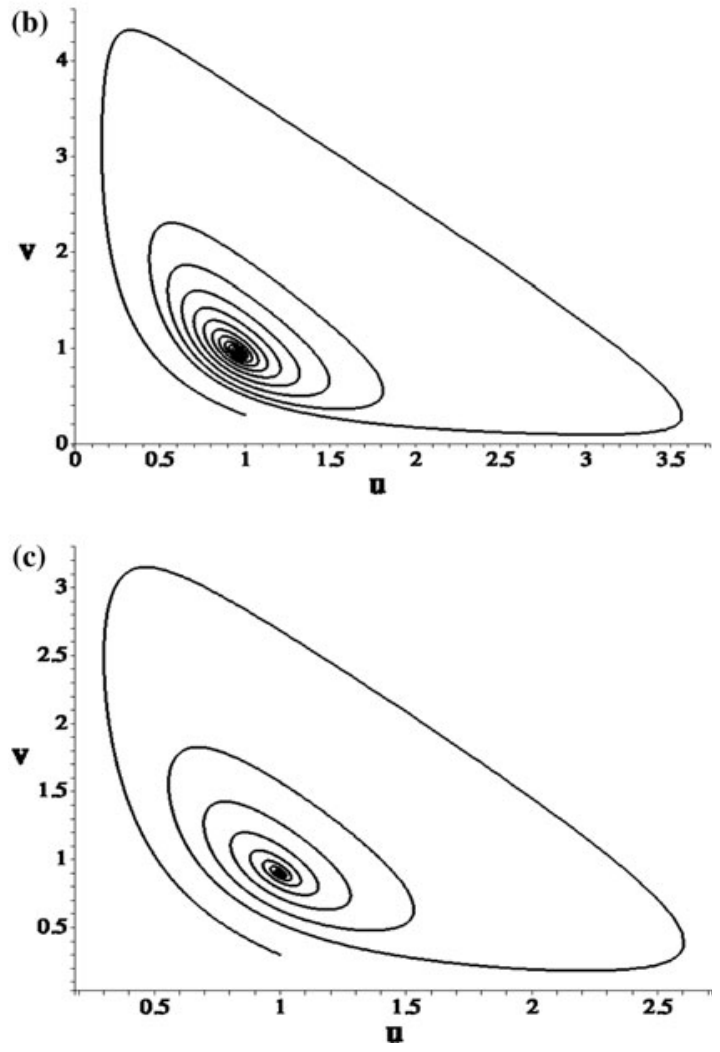

model parameter values, the matrix $M_{K}(t)$ has real eigenvalues and therefore $h_{*}=$ $h\left(t_{1}\right)$.

In Fig. 3 we present the Turing spaces corresponding to the linear and logistic growth functions respectively for each $h_{*}$ in Table 2 for fixed values of $t_{1}$. Observe that as $t_{1}$ increases, the parameter spaces shift away from the space obtained in the absence of domain growth. 

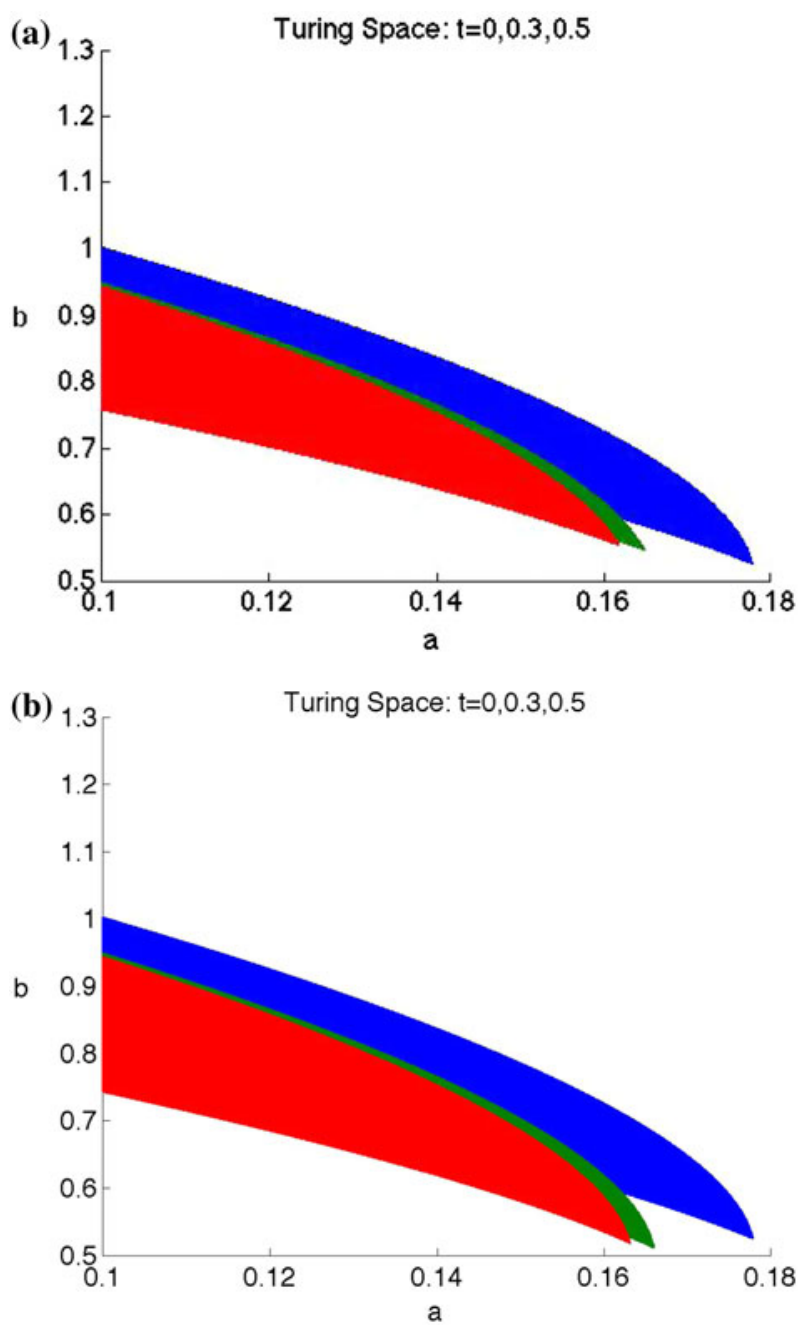

Fig. 3 Comparison between Turing spaces on continuously growing domains. The spaces at $t_{1}=0.1,0.3$ and 0.5 are superimposed for both the linear (a) and logistic (b) growth functions. The space at $t_{1}=0.1$ (middle) is the one closest to the space on a fixed domain (rightmost). As $t_{1}$ increases, the spaces shift away from the space on a fixed domain as shown in $\mathbf{c}$ for $t_{1}=0.5$ (leftmost)

\subsection{Activator-activator model: a model that violates the cross and/or pure kinetic} conditions

In this section we consider the following reaction kinetics

$$
\begin{aligned}
& f(u, v)=\delta(u-1)+(v-1)+2 \delta(v-1)^{3}, \\
& g(u, v)=-(u-1)+(v-1)+(v-1)^{2}-(v-1)^{3},
\end{aligned}
$$

where $\delta$ is a positive parameter. 
Table 2 Computing $h_{*}=h\left(t_{1}\right)$ (correct to $3 \mathrm{sig}$. figs) corresponding to the linear and logistic growth functions given $t_{1}$ and a fixed growth rate $r=0.1$

\begin{tabular}{llll}
\hline Time & Growth rate & Linear & Logistic \\
\hline$t_{1}$ & $r$ & $h_{*}$ & $h_{*}$ \\
0.1 & 0.1 & 0.198 & 0.198 \\
0.2 & 0.1 & 0.196 & 0.196 \\
0.3 & 0.1 & 0.194 & 0.194 \\
0.4 & 0.1 & 0.192 & 0.192 \\
0.5 & 0.1 & 0.191 & 0.190
\end{tabular}

The eigenvalues of $M_{K}(t)$ are real given the model parameter values chosen and hence the $h_{*}$ should be equal. Errors only occur in the third significant figure, indicating that errors are $O\left(\epsilon^{3}\right)$ which is amply accurate for our $O(\epsilon)$ accurate analysis

In the absence of domain growth, the uniform steady state is given by $(1,1)$. It can be easily shown that

$$
\begin{aligned}
\left(\begin{array}{ll}
f_{u} & f_{v} \\
g_{u} & g_{v}
\end{array}\right) & =\left.\left(\begin{array}{ll}
\delta & 1+6(v-1)^{2} \\
-1 & 1+2(v-1)-3(v-1)^{2}
\end{array}\right)\right|_{(1,1)}=\left(\begin{array}{ll}
\delta & 1 \\
-1 & 1
\end{array}\right) \\
& =\left(\begin{array}{ll}
+ & + \\
- & +
\end{array}\right)
\end{aligned}
$$

and therefore no Turing patterns will be observed since the system has neither cross nor pure kinetics. The reaction system given by Eqs. (55) and (56) is an activator-activator mechanism.

By solving the model equations (8) with reaction kinetics (55) and (56) on a onedimensional growing domain we illustrate in Fig. 4 that Turing patterns can be induced due to the incorporation of domain growth. For the simulations, we assume that $\gamma=$ $1.0, d=0.0009$ and $\delta=0.001$. Here we fix the parameters values as: the growth rate $r=0.1$ (exponential, linear and logistic growths), $m=1$ and $\kappa=2.0$ (for the logistic growth). Using the backward Euler method we compute approximate solutions with $\Delta t=10^{-4}$ and $\Delta x=10^{-2}$. We assume homogeneous Dirichlet boundary conditions. Computationally, similar results can be obtained using the Crank-Nicolson or the second order finite differentiation formula (results not shown). Furthermore, patterns can be generated also with homogeneous Neumann boundary conditions (results not shown). Initial conditions are taken as small positive perturbations of the uniform steady state. It can be observed that initially, no patterns are formed until the growing domain reaches a certain threshold whereby the inequalities (30)-(33) are satisfied. In the absence of domain growth, no Turing patterns occur. This example illustrates that the diffusively-driven instability conditions on growing domains do not require cross nor pure kinetics, but that the incorporation of domain growth and the satisfaction of the inequalities (30)-(33) is necessary for the emergence of Turing patterns. Furthermore, a sufficient condition is that there exists an excitable wavenumber. 

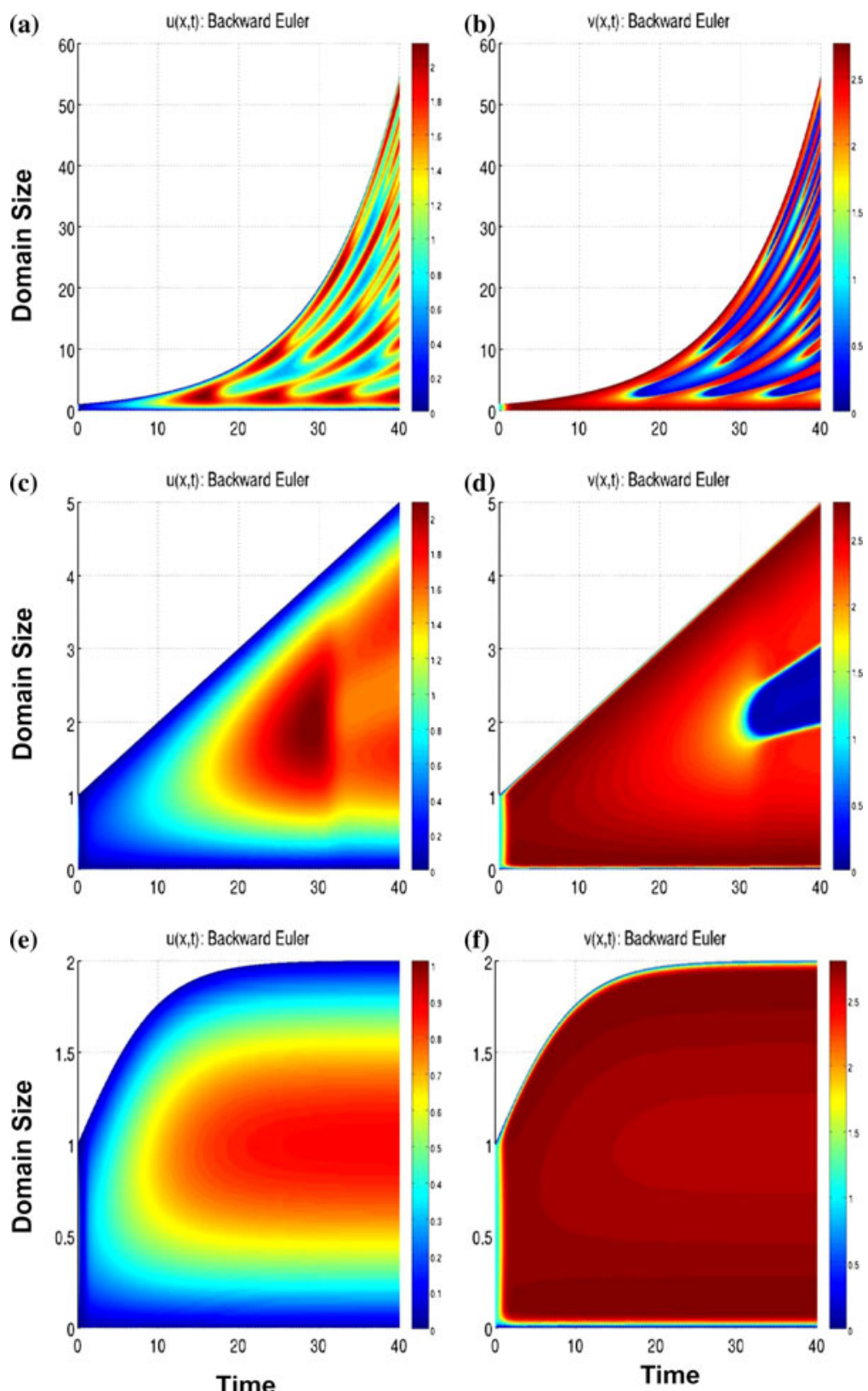

Fig. 4 Turing patterns corresponding to reaction kinetics (55)-(56) on a one-dimensional exponentially $(\mathbf{a}, \mathbf{b})$, linearly $(\mathbf{c}, \mathbf{d})$ and logistically $(\mathbf{e}, \mathbf{f})$ growing domain

Detailed observation of Fig. 4(a) and (b) reveals a mode tripling behaviour rather than the standard mode doubling behaviour. For example simultaneous peak splitting and insertion can be observed in Fig. 4(b), for $t \in[25,30]$. This mode tripling 
behaviour in one-dimension is well understood and has previously been explored (Crampin et al. 2002). In particular, it emerges from the choice of kinetics; it is not a novel feature of the non-autonomy in the pattern formation mechanism.

Similar results can be obtained with higher values up to $\delta=3$ (results not shown). Beyond $\delta=3$ patterns emerge on growing domains for these particular kinetics which do not behave in the manner of a standard diffusively-driven Turing instability. In particular, both temporal and spatial oscillations are observed rather than a steady or quasi steady spatially heterogeneous pattern.

\section{Discussion and summary}

The theoretical analysis of the role and implications of domain growth in biological pattern formation has remained largely elusive. In most cases, as the first step in considering the Turing diffusively-driven instability analysis on growing domains, the reaction-diffusion equations (RDEs) are transformed into RDEs on fixed domains, but with time-dependence in the diffusion and dilution terms (Crampin et al. 1999; Plaza et al. 2004). These non-autonomous terms however typically invalidate standard linear stability analysis via plane wave decompositions, even with the common simplification that the domain growth is assumed to be isotropic. In this paper we have presented and derived a generalisation of the classic Turing diffusively-driven instability results for the case of continuously deforming domains under the assumptions of slow growth.

Our results show that the stability conditions in the presence of growth are fundamentally different from those obtained in the absence of domain growth. To illustrate our theoretical findings we initially presented a short range activator-long range inhibitor mechanism well-known for its potential to generate Turing patterns on fixed and growing domains. Assuming exponential, linear and logistic growth profiles of the domain, we explicitly computed the Turing parameter spaces and demonstrated that the Turing spaces generally change in size and, especially, shift location within parameter space once slow growth is considered.

We also numerically investigated an activator-activator mechanism that cannot generate Turing patterns on fixed domains. The presented results explicitly illustrate our theoretical prediction that the presence of a diffusively-driven instability no longer necessitates the standard paradigm of short-range activation and long-range inhibition (Murray 2002). As we have shown, it is possible for two activators to generate a diffusively-driven instability on a growing domain. Our generalised Turing analysis also does not exclude short range inhibition and long range activation as a means to generate pattern via diffusion on growing domains.

Finally, we note that there are some similarities with regards to diffusively-driven instability conditions on fixed and growing domains. Firstly, a stable solution on a sufficiently small domain is spontaneously diffusively-driven unstable as the domain grows beyond some critical threshold, thereby satisfying the Turing conditions (30)(33). In addition a diffusively-driven instability cannot occur via a Hopf bifurcation and unequal diffusion coefficients are necessary.

The implications of our findings for models of biological pattern formation are clear. Firstly, we have shown that pattern initiation is in general sensitive to, and 
changes with, even slow domain growth. This is quite distinct from the commonly reported result that domain growth enhances the robustness of pattern selection (Plaza et al. 2004). Nonetheless, despite its effects, slow domain growth will not prohibit the Turing mechanism as a means of driving a symmetry breaking bifurcation nor will slow domain growth induce oscillations at the bifurcation, as required for biological pattern. Indeed, our most important observation is that the relaxation of the need for short range activation and long-range inhibition importantly entails that a wider range of biological morphogen pairings have the potential to induce Turing patterning on a growing domain compared to a fixed domain. It is now possible to suggest and investigate, for example, activator-activator or short-range inhibition, long-range activation as paradigms for biological pattern formation on growing domains. More generally, there exists a larger family of RDEs capable of generating diffusively-driven instability patterns in the presence of domain growth.

We have previously reported in the literature that domain growth enhances robustness of certain patterns in a Turing mechanism (Maini et al. 2002; Plaza et al. 2004). In this paper, we have shown that growth can not only induce instability in a Turing system but can also expand the range of mechanisms that can give rise to spatial patterns away from the classical short-range activation, long-range inhibition paradigm. The Turing spaces give an extensive classification of parameter values, but not growth rates. However, the growth rates for which such a result holds requires further exploration.

Acknowledgments AM would like to acknowledge Professors Georg Hetzer and Wenxian Shen (Auburn University, USA) for fruitful discussions. EAG: This publication is based on work supported in part by Award No. KUK-C1-013-04, made by King Abdullah University of Science and Technology (KAUST). PKM was partially supported by a Royal Society Wolfson Merit Award.

\section{References}

Castets V, Dulos E, Boissonade J, De Kepper P (1990) Experimental evidence of a sustained Turing-type equilibrium chemical pattern. Phys Rev Lett 64(3):2953-2956

Chaplain M, Ganesh AJ, Graham IG (2001) Spatio-temporal pattern formation on spherical surfaces: numerical simulation and application to solid tumor growth. J Math Biol 42:387-423

Connell McCluskey C (2005) A strategy for constructing Lyapunov functions for non-autonomous linear differential equations. Linear Algebra Appl 409:100-110

Crampin EJ, Gaffney EA, Maini PK (1999) Reaction and diffusion on growing domains: scenarios for robust pattern formation. Bull Math Biol 61:1093-1120

Crampin EJ, Gaffney EA, Maini PK (2002) Mode doubling and tripling in reaction-diffusion patterns on growing domains: a piecewise linear model. J Math Biol 44:107-128

Crampin EJ, Hackborn WW, Maini PK (2002) Pattern formation in reaction-diffusion models with nonuniform domain growth. Bull Math Biol 64:746-769

Edelstein-Keshet L (1988) Mathematical models in biology. Random House, New York

Gierer A, Meinhardt H (1972) A theory of biological pattern formation. Kybernetik 12:30-39

Gjorgjieva J, Jacobsen J (2007) Turing patterns on growing spheres: the exponential case. Dynamical systems and differential equations. In: Proceedings of the 6th AIMS international conference. Discrete continuous dynamical systems supplement, USA, pp 436-445

Golub GH, Van Loan CF (1996) Matrix computations. JHU Press ISBN 0801854148

Kondo S, Asai R (1995) A reaction-diffusion wave on the skin of the marine anglefish, Pomacanthus. Nature 376:765-768

Liaw SS, Yang CC, Liu RT, Hong JT (2001) Turing model patterns of lady beetles. Phys Rev E 64:041909 
Madzvamuse A (2005) Time-stepping schemes for moving grid finite elements applied to reaction-diffusion systems on fixed and growing domains. J Comp Phys 24(1):239-263

Madzvamuse A (2008) Stability analysis of reaction-diffusion systems with constant coefficients on growing domains. Int J Dyn Diff Eq 1(4):250-262

Madzvamuse A, Maini PK (2007) Velocity-induced numerical solutions of reaction-diffusion systems on fixed and growing domains. J Comp Phys 225:100-119

Madzvamuse A, Maini PK, Wathen AJ (2003) A moving grid finite element method applied to a model biological pattern generator. J Comp Phys 190:478-500

Madzvamuse A, Wathen AJ, Maini PK (2005) A moving grid finite element method for the simulation of pattern generation by Turing models on growing domains. J Sci Comp 24(2):247-262

Maini PK, Crampin EJ, Madzvamuse A, Wathen AJ, Thomas RDK (2002) Implications of domain growth in morphogenesis. In: Capaso V (ed) Mathematical modelling and computing in biology and medicine. Proceedings of the 5th European conference for mathematics and theoretical biology: conference, Milan, vol 153, pp 67-73

Maini PK, Baker RE, Chong CM (2006) The Turing model comes of molecular age, (Invited Perspective). Science 314:1397-1398

Murray JD (2002) Mathematical biology I and II, 3rd edn. Springer, Berlin

Oster AM, Bressloff PC (2006) A developmental model of ocular dominance column formation on a growing cortex. Bull Math Biol 68:73-98

Ouyang Q, Swinney HL (1991) Transition from a uniform state to hexagonal and striped Turing patterns. Nature 352:610-612

Painter KJ, Othmer HG, Maini PK (1999) Stripe formation in juvenile Pomacanthus explained by a generalized Turing mechanism with chemotaxis. Proc Natl Acad Sci 96:5549

Plaza RG, Sánchez-Garduño F, Padilla P, Barrio RA, Maini PK (2004) The effect of growth and curvature on pattern formation. J Dynam Diff Equ 16(4):1093-11214

Prigogine I, Lefever R (1968) Symmetry breaking instabilities in dissipative systems. II. J Chem Phys 48:1695-1700

Schnakenberg J (1979) Simple chemical reaction systems with limit cycle behaviour. J Theor Biol 81:389_ 400

Sick S, Reinker S, Timmer J, Schlake T (2006) WNT and DKK determine hair follicle spacing through a reaction-diffusion mechanism. Science 314:1447-1450

Solnica-Krezel L (2003) Vertebrate development: taming the nodal waves. Curr Biol 13:R7-9

Turing A (1952) The chemical basis of morphogenesis. Phil Trans R Soc Lond B 237:37-72

Varea C, Aragón JL, Barrio RA (1999) Confined Turing patterns in growing systems. Phys Rev E 60:45884592 